\title{
Towards the underlying gauge theory of the pure spinor superstring
}

\author{
Renann Lipinski Jusinskas \\ Institute of Physics of the Czech Academy of Sciences, \\ CEICO - Central European Institute for Cosmology and Fundamental Physics, \\ Na Slovance 2, 182 21, Prague, Czech Republic \\ E-mail: renannlj@fzu.cz
}

ABstract: Previous attempts to determine the worldsheet origin of the pure spinor formalism were not completely successful, but introduced important concepts that seem to be connected to its fundamental structure, e.g., emergent supersymmetry and the role of reparametrization symmetry.

In this work, a new proposal towards the underlying gauge theory of the pure spinor superstring is presented, based on an extension of Berkovits' twistor-like constraint. The gauge algebra is analyzed in detail and worldsheet reparametrization is shown to be a redundant symmetry. The master action is built with a careful account of the intrinsic gauge symmetries associated with the pure spinor constraint and a consistent gauge fixing is performed. After a field redefinition, spacetime supersymmetry emerges and the resulting action describes the pure spinor superstring.

KEYwords: Superstrings and Heterotic Strings, BRST Quantization, Topological Strings ARXIV EPRINT: 1903.10753 


\section{Contents}

1 Overview 1

2 The pure spinor superstring $\quad \mathbf{5}$

2.1 Review of the Polyakov action in the first order formalism 5

$\begin{array}{ll}2.2 & \text { The twistor-like constraint in the first order formalism }\end{array}$

2.3 A new model with constrained anticommuting spinors 8

$\begin{array}{ll}2.4 \text { The pure spinor master action } & 11\end{array}$

$\begin{array}{ll}2.5 \text { Gauge fixing } & 17\end{array}$

$\begin{array}{lll}2.6 & \text { Field redefinition and emergent spacetime supersymmetry } & 22\end{array}$

$\begin{array}{llr}3 & \text { Final remarks } & 25\end{array}$

$\begin{array}{ll}\text { A Partial gauge fixing of the pure spinor symmetries } & 26\end{array}$

$\begin{array}{lll}\text { B The } \mathrm{U}(1)_{R} \times \mathrm{U}(1)_{L} \text { sector } & 28\end{array}$

C A non-minimal formalism with fundamental $(b, c)$ ghosts 29

D The sectorized and ambitwistor pure spinor superstrings 31

\section{Overview}

The pure spinor formalism of the superstring was introduced by Berkovits almost two decades ago [1]. Since then, it has been studied and explored in many different aspects, taking advantage of its symmetry preserving character and bosonic string-like amplitude prescription. These aspects range from the impressive 3-loop computation of scattering amplitudes of [2] or the recent $N$-point 1-loop results of [3-5], to the investigation of the quantization of the superstring in the $A d S_{5} \times S^{5}$ background (see [6] for a review and references therein) or to the analysis of supersymmetry breaking effects in the superstring [7].

There is abundant evidence that the pure spinor superstring is related to the spinning string $[8,9]$ and to the Green-Schwarz superstring [10, 11]. Scattering amplitudes computed in the pure spinor superstring were shown to be equivalent to the spinning string amplitudes up to two loops [12]. Furthermore, it has been shown in [13] that the pure spinor cohomology in the light-cone gauge describes the usual physical spectrum of the superstring. Later on this equivalence was explored in [14] and more recently in [15], where a combination of field redefinitions and similarity transformations helped to identify the Green-Schwarz and the pure spinor superstrings. In [16], the DDF-like structure of the pure spinor cohomology was finally made explicit. From another perspective, superembedding techniques (see [17] for a review and references therein) seem to provide a fertile 
ground for exploring the classical equivalence between the different superstring formalisms (e.g. $[18,19])$. The superembedding origin of the pure spinor description of the heterotic superstring was demonstrated in [20].

However, the pure spinor formalism lacks a fundamental worldsheet description, meaning (1) a two-dimensional reparametrization invariant gauge theory which upon quantization concretely leads to its characteristic BRST structure and (2) a first principles derivation of the pure spinor constraint itself. The goal of this work is to present a possible resolution for the point (1), but still assuming that the pure spinor is a fundamental variable.

The twistor-like constraint. The first step to understand the gauge structure of the pure spinor formalism from a more fundamental point of view was taken in [21] with the introduction of the twistor-like constraint

$$
\begin{aligned}
H_{\alpha} & \equiv P_{m}\left(\gamma^{m} \lambda\right)_{\alpha}, \\
& =0
\end{aligned}
$$

where $P_{m}$ is the canonical conjugate of the target-space coordinate $X^{m}$, with $m=0, \ldots, 9$, $\gamma_{\alpha \beta}^{m}$ denotes the chiral blocks of the Dirac matrices, with $\alpha=1, \ldots, 16$, and $\lambda^{\alpha}$ is a pure spinor variable satisfying

$$
\left(\lambda \gamma^{m} \lambda\right)=0
$$

The novel feature of this approach was that $\lambda^{\alpha}$ appeared as a fundamental variable in the worldline/worldsheet ${ }^{1}$ and the superpartners of $X^{m}$, denoted by $\theta^{\alpha}$, entered the formalism as ghost fields associated to the gauge symmetry generated by (1.1). In this model, supersymmetry is an emergent feature related to a ghost twisting operation on the gauge fixed action. However, the gauge symmetries related to the pure spinor constraint were not completely considered in this approach, leading to an incorrect description of the ghost fields.

A new attempt to quantize the twistor-like constraint was made in [22], with a different mechanism for the emergence of spacetime supersymmetry. The problem of this proposal was an overconstrained action which ultimately leads to a trivialization of the model.

This flaw was later corrected in [23], where a new gauge theory was proposed to explain the origin of the pure spinor formalism. Berkovits' first order action can be cast as

$$
\begin{aligned}
S_{B}= & \int d \tau d \sigma\left\{P_{m} \partial_{\tau} X^{m}+w_{\alpha} \nabla_{\tau} \lambda^{\alpha}+\hat{w}_{\hat{\alpha}} \hat{\nabla}_{\tau} \hat{\lambda}^{\hat{\alpha}}+K_{\alpha} \nabla_{\sigma} \lambda^{\alpha}+\hat{K}_{\hat{\alpha}} \hat{\nabla}_{\sigma} \hat{\lambda}^{\hat{\alpha}}\right. \\
& \left.-\frac{1}{2} L^{\alpha}\left(\gamma^{m} \lambda\right)_{\alpha}\left(P_{m}+\partial_{\sigma} X_{m}\right)-\frac{1}{2} \hat{L}^{\hat{\alpha}}\left(\gamma^{m} \hat{\lambda}\right)_{\hat{\alpha}}\left(P_{m}-\partial_{\sigma} X_{m}\right)\right\} .
\end{aligned}
$$

Here, $\tau$ and $\sigma$ denote the worldsheet coordinates and hatted and unhatted spinors are related to the usual left and right-moving variables. The Lagrange multipliers $\left\{L^{\alpha}, \hat{L}^{\hat{\alpha}}\right\}$ impose the twistor-like constraints and $\left\{K_{\alpha}, \hat{K}_{\hat{\alpha}}\right\}$, as a consequence of the gauge algebra, impose the constraints $\nabla_{\sigma} \lambda^{\alpha}=\hat{\nabla}_{\sigma} \hat{\lambda}^{\hat{\alpha}}=0$. The covariant derivatives $\nabla$ and $\hat{\nabla}$ are

\footnotetext{
${ }^{1}$ Twistor-like variables arise naturally using superembedding techniques and the first appearance of pure spinors in this context was in [19].
} 
composed by gauge fields, $A$ and $\hat{A}$, associated to local scale symmetries for $\lambda^{\alpha}$ and $\hat{\lambda}^{\hat{\alpha}}$, which effectively convert them into projective pure spinors. Although not explicitly, $S_{B}$ is invariant under worldsheet reparametrization.

One of the fundamental ideas introduced in these works [21-23] is that worldsheet reparametrization is a redundant gauge symmetry (in the sense that it can be removed by a gauge-for-gauge transformation). With this in mind, the action (1.3) is already incomplete as the absence of a kinetic term for the gauge fields of the scaling symmetry prevents the existence of a gauge-for-gauge symmetry connecting the twistor-like constraints and reparametrization. Aside from this fact, the gauge symmetries due to the pure spinor constraint imply a constrained ghost system associated to the twistor-like constraint, such that the gauge fixed action cannot be spacetime supersymmetric. Observe that the action (1.3) is invariant under the gauge transformation

$$
\delta L^{\alpha}=f \lambda^{\alpha}+f^{m n}\left(\gamma_{m n} \lambda\right)^{\alpha},
$$

where $\left\{f, f_{m n}\right\}$ are the gauge parameters and $\gamma^{m n}=\frac{1}{2}\left(\gamma^{m} \gamma^{n}-\gamma^{n} \gamma^{m}\right)$. In the gauge fixing procedure, this gauge symmetry appears as a constraint on the antighost of the twistor-like symmetry, $\pi_{\alpha}$, given by

$$
\left(\lambda \gamma^{m} \gamma^{n} \pi\right)=0
$$

In turn, it implies that only five components of the associated ghost, $\theta^{\alpha}$, are physical. Therefore, the phase space of the action (1.3) should be extended if the twistor-like constraint is to be part of a spacetime supersymmetric theory.

It is interesting to note, however, that all these features were in some sense convergent, leading to important concepts that seem to be connected to the gauge structure behind the pure spinor superstring, in particular the role of worldsheet reparametrization and the emergence of spacetime supersymmetry from the ghost sector.

The extended action. A simple way to understand the physical meaning of the twistorlike constraint is to look at its worldline version, as the massless particle can be viewed as the zero length limit of the string. Consider the constraint equation (1.1), but now with a projective pure spinor. In a Wick-rotated construction, the $\mathrm{SO}(10)$ spinor $H_{\alpha}$ can be decomposed in terms of $\mathrm{U}(5)$ components such that

$$
\bar{p}^{a}+\gamma^{a b} p_{b}=0
$$

correspond to the independent components of $H_{\alpha}=0$. Here, $a=1, \ldots, 5$ denotes $\mathrm{U}(5)$ vector indices, $P_{m}=\left\{\bar{p}^{a}, p_{a}\right\}$ and $\gamma^{a b}=-\gamma^{b a}$ corresponds to the $\mathrm{U}(5)$ parametrization of the projective pure spinor. Equation (1.6) has a clear interpretation as any solution of the massless constraint $P^{m} P_{m}=0$ can be put in this form for a dynamical $\gamma^{a b}$.

The difference between the covariant form (1.1) and (1.6) is basically the scale symmetry introduced by Berkovits in [23]. Both of them were recently investigated by the author in [24]. In order to obtain the pure spinor superparticle from first principles, Berkovits' model was then extended with a constrained anticommuting spinor together with an additional fermionic gauge symmetry. Now, this idea will be generalized to the worldsheet 
with the proposal of the action

$$
\begin{aligned}
S_{0}= & \int d \tau d \sigma\left\{P_{m} \partial_{\tau} X^{m}-\frac{1}{4 \mathcal{T}}\left(L \gamma_{m} \lambda\right)\left(P^{m}+\mathcal{T} \partial_{\sigma} X^{m}\right)-\frac{1}{4 \mathcal{T}}\left(\hat{L} \gamma_{m} \hat{\lambda}\right)\left(P^{m}-\mathcal{T} \partial_{\sigma} X^{m}\right)\right\} \\
& +\int d \tau d \sigma\left\{w_{\alpha}^{i} \nabla_{i} \lambda^{\alpha}+B \epsilon^{i j} \partial_{i} A_{j}+p_{\alpha}^{i} \partial_{i} \xi^{\alpha}+\chi_{i} \lambda^{\alpha} p_{\alpha}^{i}-\Sigma \epsilon^{i j} \nabla_{i} \chi_{j}\right\} \\
& +\int d \tau d \sigma\left\{\hat{w}_{\hat{\alpha}}^{i} \hat{\nabla}_{i} \hat{\lambda}^{\hat{\alpha}}+\hat{B} \epsilon^{i j} \partial_{i} \hat{A}_{j}+\hat{p}_{\hat{\alpha}}^{i} \partial_{i} \hat{\xi}^{\hat{\alpha}}+\hat{\chi}_{i} \hat{\lambda}^{\hat{\alpha}} \hat{p}_{\hat{\alpha}}^{i}-\hat{\Sigma} \epsilon^{i j} \hat{\nabla}_{i} \hat{\chi}_{j}\right\}
\end{aligned}
$$

Here, $\{i, j\}$ denote the worldsheet directions $\tau$ and $\sigma$, and $\mathcal{T}$ is the string tension. The spinors $\xi^{\alpha}$ and $\hat{\xi}^{\hat{\alpha}}$, with conjugates $p_{\alpha}^{i}$ and $\hat{p}_{\hat{\alpha}}^{i}$, satisfy

$$
\left(\lambda \gamma^{m} \xi\right)=\left(\hat{\lambda} \gamma^{m} \hat{\xi}\right)=0 .
$$

The motivation for introducing the constrained spinors $\xi^{\alpha}$ and $\hat{\xi}^{\hat{\alpha}}$ is that they should suplement the degrees of freedom from the ghosts of the twistor-like symmetry, combining into the superpartners of $X^{m}$. The fermionic symmetry in $S_{0}$ is generated by the current $\lambda^{\alpha} p_{\alpha}^{i}$, with Lagrange multiplier $\chi_{i}$. The fields $B$ and $\Sigma$ effectively work as conjugates to the gauge field of the scale symmetry $\left(A_{i}\right)$ and its fermionic partner $\left(\chi_{i}\right)$, respectively. They can be interpreted as Lagrange multipliers for a zero curvature condition on the gauge fields, $\epsilon^{i j} \partial_{i} A_{j}=\epsilon^{i j} \nabla_{i} \chi_{j}=0$, with $\epsilon^{i j}=-\epsilon^{j i}\left(\epsilon_{i j}=-\epsilon_{j i}\right)$ and $\epsilon^{\sigma \tau}=\epsilon_{\tau \sigma}=1$. The hatted variables have an analogous description.

The reparametrization invariant action (1.7) has a rich gauge structure. Its gauge algebra is onshell reducible and reparametrization symmetry can be consistently overlooked in the quantization process due to the simple form of the gauge-for-gauge symmetries. Still, the Batalin-Vilkovisky formalism seems to be the most adequate for the quantization of this model, because it provides a systematic way to analyze the gauge symmetries related to the pure spinor constraints. The extra fermionic symmetry of the action (1.7) does not have a clear physical interpretation but it is ultimately related to the emergence of spacetime supersymmetry in the gauge fixed action, which can be cast as

$$
S=\int d \tau d \sigma\left\{\frac{\mathcal{T}}{2} \partial_{+} X_{m} \partial_{-} X^{m}+w_{\alpha} \partial_{-} \lambda^{\alpha}+p_{\alpha} \partial_{-} \theta^{\alpha}+\hat{w}_{\hat{\alpha}} \partial_{+} \hat{\lambda}^{\hat{\alpha}}+\hat{p}_{\hat{\alpha}} \partial_{+} \hat{\theta}^{\hat{\alpha}}\right\}
$$

where $\partial_{ \pm}=\partial_{\tau} \pm \partial_{\sigma}$, corresponding to the usual superstring action in the pure spinor formalism. In this action, $\theta^{\alpha}$ and $\hat{\theta}^{\hat{\alpha}}$, the superpartners of the target space coordinate $X^{m}$, are composed by the ghosts associated to the twistor-like symmetry complemented with the constrained spinors $\xi^{\alpha}$ and $\hat{\xi}^{\hat{\alpha}}$. This composition is only possible due to the existence of the scalar ghosts $\gamma$ and $\hat{\gamma}$ associated to the new fermionic symmetry in (1.7). They are assumed to be everywhere non-vanishing in the worldsheet, effectively acting as ghost number twisting operators and turning all the spacetime spinors neutral under scale transformations. This is the mechanism behind the conversion of the pure spinors $\lambda^{\alpha}$ and $\hat{\lambda}^{\hat{\alpha}}$ into ghost variables.

Plan of the paper. Section 2 presents in detail a first principles derivation of the pure spinor superstring. Subsection 2.1 quickly reviews the first order formulation of 
the Polyakov action and subsection 2.2 describes the connection between the twistorlike constraints and worldsheet reparametrization, motivating Berkovits' model [23]. In subsection 2.3, the action (1.7) is introduced with a thorough analysis of its gauge and gauge-for-gauge symmetries. Subsection 2.4 describes the construction of the pure spinor master action, while subsection 2.5 presents its gauge fixing. Subsection 2.6 closes the section with the description of the emergence of spacetime supersymmetry leading to the pure spinor superstring. Section 3 contains some concluding remarks and possible directions to follow.

Throughout this work, special attention has been devoted to displaying auxiliary equations in the intermediate steps, so most computations can be promptly reproduced. The appendices consist of several complements and by-products of the main text. Appendix A shows how the partial gauge fixing of the pure spinor symmetries leads to the unconstrained spinor $\theta^{\alpha}$, the superpartner of the target space coordinates $X^{m}$. Appendix B presents some properties of the $\mathrm{U}(1)_{R} \times \mathrm{U}(1)_{L}$ sector constituted by the ghost fields related to the scaling and fermionic symmetries, respectively with parameters $\{\Omega, \hat{\Omega}\}$ and $\{\gamma, \hat{\gamma}\}$. Appendix $\mathrm{C}$ describes a non-minimal pure spinor superstring with fundamental $(b, c)$ ghosts, as suggested in [22]. Finally, appendix D presents the sectorized and the ambitwistor string in the pure spinor formalism as coming from a singular gauge fixing of the action (1.7).

\section{The pure spinor superstring}

In this section, the connection between worldsheet reparametrization and the twistor-like constraint will be investigated, with a concrete proposal for the underlying gauge theory of the pure spinor superstring.

\subsection{Review of the Polyakov action in the first order formalism}

The Polyakov action is given by

$$
S_{P}=\frac{\mathcal{T}}{2} \int d^{2} \sigma \sqrt{-g}\left\{g^{i j} \partial_{i} X^{m} \partial_{j} X_{m}\right\}
$$

where $\mathcal{T}$ is the string tension, $g^{i j}$ is the worldsheet metric (with inverse $g_{i j}$ ) and $g=\operatorname{det}\left(g_{i j}\right)$. When expanded in components $(i=\tau, \sigma)$, with $\tau$ denoting the worldsheet time and $\sigma$ parametrizing the string length, $S_{P}$ is rewritten as

$$
S_{P}=\frac{\mathcal{T}}{2} \int d \tau d \sigma \sqrt{-g}\left\{g^{\tau \tau} \partial_{\tau} X^{m} \partial_{\tau} X_{m}+2 g^{\tau \sigma} \partial_{\tau} X^{m} \partial_{\sigma} X_{m}+g^{\sigma \sigma} \partial_{\sigma} X^{m} \partial_{\sigma} X_{m}\right\} .
$$

The canonical conjugate of the target-space coordinate $X^{m}$ is easily determined to be

$$
\begin{aligned}
P_{m} & \equiv \frac{\delta S}{\delta \partial_{\tau} X^{m}}, \\
& =\mathcal{T} \sqrt{-g}\left\{g^{\tau \tau} \partial_{\tau} X_{m}+g^{\tau \sigma} \partial_{\sigma} X_{m}\right\}
\end{aligned}
$$

leading to the Hamiltonian

$$
\begin{aligned}
H & \equiv P_{m} \partial_{\tau} X^{m}-\frac{\mathcal{T}}{2} \sqrt{-g} g^{i j} \partial_{i} X^{m} \partial_{j} X_{m} \\
& =\frac{1}{2 \mathcal{T} g^{\tau \tau} \sqrt{-g}}\left(P_{m} P^{m}+\mathcal{T}^{2} \partial_{\sigma} X_{m} \partial_{\sigma} X^{m}\right)-\frac{g^{\tau \sigma}}{g^{\tau \tau}} P_{m} \partial_{\sigma} X^{m}
\end{aligned}
$$


In the first order formulation, the Polyakov action takes the form

$$
\tilde{S}_{P}=\int d \tau d \sigma\left\{P_{m} \partial_{\tau} X^{m}-\frac{1}{2 \mathcal{T} g^{\tau \tau} \sqrt{-g}}\left(P_{m} P^{m}+\mathcal{T}^{2} \partial_{\sigma} X_{m} \partial_{\sigma} X^{m}\right)+\frac{g^{\tau \sigma}}{g^{\tau \tau}} P_{m} \partial_{\sigma} X^{m}\right\}
$$

which is equivalent onshell to $S_{P}$, cf. equation (2.3). Observe that the only dependence on the worldsheet metric appears now in the form of Lagrange multipliers. In fact, by defining the Weyl invariant operators

$$
e_{ \pm} \equiv \frac{1}{g^{\tau \tau} \sqrt{-g}} \mp \frac{g^{\tau \sigma}}{g^{\tau \tau}}
$$

the action $\tilde{S}_{P}$ is more symmetrically rewritten as

$$
\begin{aligned}
\tilde{S}_{P}= & \int d \tau d \sigma\left\{P_{m} \partial_{\tau} X^{m}-\frac{1}{4 \mathcal{T}} e_{+}\left(P_{m}+\mathcal{T} \partial_{\sigma} X_{m}\right)\left(P^{m}+\mathcal{T} \partial_{\sigma} X^{m}\right)\right. \\
& \left.-\frac{1}{4 \mathcal{T}} e_{-}\left(P_{m}-\mathcal{T} \partial_{\sigma} X_{m}\right)\left(P^{m}-\mathcal{T} \partial_{\sigma} X^{m}\right)\right\} .
\end{aligned}
$$

The equations of motion for $P_{m}, X^{m}$ and $e_{ \pm}$are respectively given by

$$
\begin{aligned}
\partial_{\tau} X^{m}-\frac{1}{2 \mathcal{T}}\left[e_{+}\left(P^{m}+\mathcal{T} \partial_{\sigma} X^{m}\right)+e_{-}\left(P^{m}-\mathcal{T} \partial_{\sigma} X^{m}\right)\right] & =0 \\
\partial_{\tau} P_{m}-\frac{1}{2} \partial_{\sigma}\left[e_{+}\left(P_{m}+\mathcal{T} \partial_{\sigma} X_{m}\right)-e_{-}\left(P_{m}-\mathcal{T} \partial_{\sigma} X_{m}\right)\right] & =0 \\
\left(P_{m} \pm \mathcal{T} \partial_{\sigma} X_{m}\right)\left(P^{m} \pm \mathcal{T} \partial_{\sigma} X^{m}\right) & =0
\end{aligned}
$$

and the action (2.7) is invariant under the gauge transformations

$$
\begin{aligned}
\delta X^{m}= & c^{i} \partial_{i} X^{m}-\frac{1}{2 \mathcal{T}} a^{+}\left(P^{m}+\mathcal{T} \partial_{\sigma} X^{m}\right)-\frac{1}{2 \mathcal{T}} a^{-}\left(P^{m}-\mathcal{T} \partial_{\sigma} X^{m}\right), \\
\delta P_{m}= & \partial_{i}\left(c^{i} P_{m}\right)-P_{m} \partial_{\tau} c^{\tau}+\frac{1}{2} \partial_{\sigma} c^{\tau}\left[\left(P_{m}+\mathcal{T} \partial_{\sigma} X_{m}\right) e_{+}-\left(P_{m}-\mathcal{T} \partial_{\sigma} X_{m}\right) e_{-}\right] \\
& -\frac{1}{2} \partial_{\sigma}\left[a^{+}\left(P^{m}+\mathcal{T} \partial_{\sigma} X^{m}\right)-a^{-}\left(P^{m}-\mathcal{T} \partial_{\sigma} X^{m}\right)\right] \\
\delta e_{+}= & \partial_{i}\left(c^{i} e_{+}\right)-2\left(\partial_{\sigma} c^{\sigma}\right) e_{+}+\partial_{\tau} c^{\sigma}-e_{+}^{2} \partial_{\sigma} c^{\tau}-\partial_{\tau} a^{+}-a^{+} \partial_{\sigma} e_{+}+e_{+} \partial_{\sigma} a^{+}, \\
\delta e_{-}= & \partial_{i}\left(c^{i} e_{-}\right)-2\left(\partial_{\sigma} c^{\sigma}\right) e_{-}-\partial_{\tau} c^{\sigma}+e_{-}^{2} \partial_{\sigma} c^{\tau}-\partial_{\tau} a^{-}+a^{-} \partial_{\sigma} e_{-}-e_{-} \partial_{\sigma} a^{-} .
\end{aligned}
$$

In addition to worldsheet reparametrization symmetry, with parameter $c^{i}$, the action $\tilde{S}_{P}$ is also invariant under the gauge transformations parametrized by $a^{ \pm}$. However, these gauge symmetries are not irreducible. To see this, consider the gauge-for-gauge transformations

$$
\begin{aligned}
\delta^{\prime} c^{i} & =\phi^{i}, \\
\delta^{\prime} a^{ \pm} & =\phi^{\tau} e_{ \pm} \pm \phi^{\sigma},
\end{aligned}
$$

with parameter $\phi^{i}$. It is then straightforward to show that the gauge transformations (2.9) are invariant up to equations of motion:

$$
\begin{aligned}
\delta^{\prime}\left[\delta X^{m}\right] & =\phi^{\tau}\left\{\partial_{\tau} X^{m}-\frac{1}{2 \mathcal{T}}\left[e_{+}\left(P^{m}+\mathcal{T} \partial_{\sigma} X^{m}\right)+e_{-}\left(P^{m}-\mathcal{T} \partial_{\sigma} X^{m}\right)\right]\right\}, \\
\delta^{\prime}\left[\delta P_{m}\right] & =\phi^{\tau}\left\{\partial_{\tau} P_{m}-\frac{1}{2} \partial_{\sigma}\left[e_{+}\left(P_{m}+\mathcal{T} \partial_{\sigma} X_{m}\right)-e_{-}\left(P_{m}-\mathcal{T} \partial_{\sigma} X_{m}\right)\right]\right\}, \\
\delta^{\prime}\left[\delta e_{+}\right] & =0 \\
\delta^{\prime}\left[\delta e_{-}\right] & =0 .
\end{aligned}
$$


Therefore, worldsheet reparametrization is equivalent to the symmetries generated by

$$
H^{ \pm} \equiv\left(P_{m} \pm \mathcal{T} \partial_{\sigma} X_{m}\right)\left(P^{m} \pm \mathcal{T} \partial_{\sigma} X^{m}\right)
$$

\subsection{The twistor-like constraint in the first order formalism}

In [23], Berkovits proposed the twistor-like constraints

$$
\begin{aligned}
H_{\alpha} & \equiv\left(P_{m}+\mathcal{T} \partial_{\sigma} X_{m}\right)\left(\gamma^{m} \lambda\right)_{\alpha}, \\
\hat{H}_{\hat{\alpha}} & \equiv\left(P_{m}-\mathcal{T} \partial_{\sigma} X_{m}\right)\left(\gamma^{m} \hat{\lambda}\right)_{\hat{\alpha}},
\end{aligned}
$$

as part of the fundamental gauge algebra behind the pure spinor superstring, where $\lambda^{\alpha}$ and $\hat{\lambda}^{\hat{\alpha}}$ are bosonic spinors satisfying the pure spinor condition

$$
\begin{aligned}
& \left(\lambda \gamma^{m} \lambda\right)=0 \\
& \left(\hat{\lambda} \gamma^{m} \hat{\lambda}\right)=0 .
\end{aligned}
$$

The key idea here is that $H^{ \pm}$in $(2.12)$ can be rewritten as

$$
\begin{aligned}
& H^{+}=\left(P_{m}+\mathcal{T} \partial_{\sigma} X_{m}\right) \frac{\left(\Lambda \gamma^{m}\right)^{\alpha}}{(\Lambda \lambda)} H_{\alpha}, \\
& H^{-}=\left(P_{m}-\mathcal{T} \partial_{\sigma} X_{m}\right) \frac{\left(\gamma^{m} \hat{\Lambda}\right)^{\hat{\alpha}}}{(\hat{\Lambda} \hat{\lambda})} \hat{H}_{\hat{\alpha}},
\end{aligned}
$$

for any constant $\Lambda_{\alpha}$ and $\hat{\Lambda}_{\hat{\alpha}}$ with non-vanishing $(\Lambda \lambda)$ and $(\hat{\Lambda} \hat{\lambda})$.

The first order Polyakov's action (2.7) can be covariantly modified with the introduction of the twistor-like constraints (2.13). In order to do that, $\lambda^{\alpha}$ and $\hat{\lambda}^{\hat{\alpha}}$ have to be made dynamical. In addition, Berkovits proposed in [23] the use of projective pure spinors which can be achieved by endowing $\lambda^{\alpha}$ and $\hat{\lambda}^{\hat{\alpha}}$ with a scaling symmetry. The resulting action is

$$
\begin{aligned}
S_{B}^{\prime}= & \int d \tau d \sigma\left\{P_{m} \partial_{\tau} X^{m}+w_{\alpha}^{i} \partial_{i} \lambda^{\alpha}+\hat{w}_{\hat{\alpha}}^{i} \partial_{i} \hat{\lambda}^{\hat{\alpha}}+A_{i} \lambda^{\alpha} w_{\alpha}^{i}+\hat{A}_{i} \hat{\lambda}^{\hat{\alpha}} \hat{w}_{\hat{\alpha}}^{i}\right\} \\
& -\frac{1}{4 \mathcal{T}} \int d \tau d \sigma\left\{\left(L \gamma_{m} \lambda\right)\left(P^{m}+\mathcal{T} \partial_{\sigma} X^{m}\right)+e_{+}\left(P_{m}+\mathcal{T} \partial_{\sigma} X_{m}\right)\left(P^{m}+\mathcal{T} \partial_{\sigma} X^{m}\right)\right. \\
& \left.+\left(\hat{L} \gamma_{m} \hat{\lambda}\right)\left(P^{m}-\mathcal{T} \partial_{\sigma} X^{m}\right)+e_{-}\left(P_{m}-\mathcal{T} \partial_{\sigma} X_{m}\right)\left(P^{m}-\mathcal{T} \partial_{\sigma} X^{m}\right)\right\}
\end{aligned}
$$

where $\left\{L^{\alpha}, \hat{L}^{\hat{\alpha}}\right\}$ are the Lagrange multipliers for the constraints $(2.13)$ and $\left\{A_{i}, \hat{A}_{i}\right\}$ are the gauge fields for the scaling symmetry generated by $\lambda^{\alpha} w_{\alpha}^{i}$ and $\hat{\lambda}^{\hat{\alpha}} \hat{w}_{\hat{\alpha}}^{i}$. Due to the identification (2.15), a field shift in $L^{\alpha}$ and $\hat{L}^{\hat{\alpha}}$ can absorb $e_{+}$and $e_{-}$, leading to Berkovits' action,

$$
\begin{aligned}
S_{B}= & \int d \tau d \sigma\left\{P_{m} \partial_{\tau} X^{m}+w_{\alpha}^{i} \nabla_{i} \lambda^{\alpha}+\hat{w}_{\hat{\alpha}}^{i} \hat{\nabla}_{i} \hat{\lambda}^{\hat{\alpha}}\right\} \\
& -\frac{1}{4 \mathcal{T}} \int d \tau d \sigma\left\{\left(L \gamma_{m} \lambda\right)\left(P^{m}+\mathcal{T} \partial_{\sigma} X^{m}\right)+\left(\hat{L} \gamma_{m} \hat{\lambda}\right)\left(P^{m}-\mathcal{T} \partial_{\sigma} X^{m}\right)\right\}
\end{aligned}
$$

The gauge fields $\left\{A_{i}, \hat{A}_{i}\right\}$ now appear through the covariant derivatives $\left\{\nabla_{i}, \hat{\nabla}_{i}\right\}$. Observe also that all the dependence on the worldsheet metric is concentrated in the Lagrange multipliers $\left\{L^{\alpha}, \hat{L}^{\hat{\alpha}}\right\}$ and this has to be taken into account during the gauge fixing process. 


\subsection{A new model with constrained anticommuting spinors}

Based on the worldline results of [24], it is straightforward to generalize the action (2.17) to

$$
\begin{aligned}
S_{0}= & \int d \tau d \sigma\left\{P_{m} \partial_{\tau} X^{m}+w_{\alpha}^{i} \nabla_{i} \lambda^{\alpha}+\hat{w}_{\hat{\alpha}}^{i} \hat{\nabla}_{i} \hat{\lambda}^{\hat{\alpha}}\right\} \\
& -\frac{1}{4 \mathcal{T}} \int d \tau d \sigma\left\{\left(L \gamma_{m} \lambda\right)\left(P^{m}+\mathcal{T} \partial_{\sigma} X^{m}\right)+\left(\hat{L} \gamma_{m} \hat{\lambda}\right)\left(P^{m}-\mathcal{T} \partial_{\sigma} X^{m}\right)\right\} \\
& +\int d \tau d \sigma\left\{p_{\alpha}^{i} \partial_{i} \xi^{\alpha}+B \epsilon^{i j} \partial_{i} A_{j}+\hat{p}_{\hat{\alpha}}^{i} \partial_{i} \hat{\xi}^{\hat{\alpha}}+\hat{B} \epsilon^{i j} \partial_{i} \hat{A}_{j}\right\} \\
& +\int d \tau d \sigma\left\{\chi_{i} \lambda^{\alpha} p_{\alpha}^{i}-\Sigma \epsilon^{i j} \nabla_{i} \chi_{j}+\hat{\chi}_{i} \hat{\lambda}^{\hat{\alpha}} \hat{p}_{\hat{\alpha}}^{i}-\hat{\Sigma} \epsilon^{i j} \hat{\nabla}_{i} \hat{\chi}_{j}\right\} .
\end{aligned}
$$

There are two guiding principles that led to the proposed action $S_{0},(1)$ the extension of the phase space with the inclusion of constrained anticommuting spinors, $\xi^{\alpha}$ and $\hat{\xi}^{\hat{\alpha}}$, satisfying

$$
\begin{aligned}
& \left(\lambda \gamma^{m} \xi\right)=0, \\
& \left(\hat{\lambda} \gamma^{m} \hat{\xi}\right)=0,
\end{aligned}
$$

together with two fermionic symmetries generated by $\lambda^{\alpha} p_{\alpha}^{i}$ and $\hat{\lambda}^{\hat{\alpha}} \hat{p}_{\hat{\alpha}}^{i}$; and (2) the introduction of zero curvature conditions on the gauge fields $\left\{A_{i}, \chi_{i}, \hat{A}_{i}, \hat{\chi}_{i}\right\}$ through the Lagrange multipliers $\{B, \Sigma, \hat{B}, \hat{\Sigma}\}$, which enables the extension of the gauge algebra of the model with the inclusion of gauge-for-gauge symmetries connecting worldsheet reparametrization and the twistor-like symmetries.

The equations of motion obtained from the action (2.18) can be summarized as

$$
\begin{aligned}
\partial_{\tau} X^{m}-\frac{1}{4 \mathcal{T}}\left(L \gamma^{m} \lambda\right)-\frac{1}{4 \mathcal{T}}\left(\hat{L} \gamma^{m} \hat{\lambda}\right) & =0, \\
\partial_{\tau} P_{m}-\frac{1}{4} \partial_{\sigma}\left(L \gamma_{m} \lambda\right)+\frac{1}{4} \partial_{\sigma}\left(\hat{L} \gamma_{m} \hat{\lambda}\right) & =0, \\
\nabla_{i} \lambda^{\alpha} & =0, \\
\nabla_{i} w_{\alpha}^{i}+\frac{1}{4 \mathcal{T}}\left(P^{m}+\mathcal{T} \partial_{\sigma} X^{m}\right)\left(\gamma_{m} L\right)_{\alpha}-\chi_{i} p_{\alpha}^{i} & =0, \\
\lambda^{\alpha} w_{\alpha}^{i}+\epsilon^{i j}\left(\Sigma \chi_{j}+\partial_{j} B\right) & =0, \\
\partial_{i} \xi^{\alpha}-\chi_{i} \lambda^{\alpha} & =0, \\
\partial_{i} p_{\alpha}^{i} & =0, \\
\epsilon^{i j} \partial_{i} A_{j} & =0, \\
\left(P^{m}+\mathcal{T} \partial_{\sigma} X^{m}\right)\left(\gamma_{m} \lambda\right)_{\alpha} & =0, \\
\lambda^{\alpha} p_{\alpha}^{i}+\epsilon^{i j} \nabla_{j} \Sigma & =0, \\
\epsilon^{i j} \nabla_{i} \chi_{j} & =0, \\
\hat{\nabla}_{i} \hat{\lambda}^{\hat{\alpha}} & =0, \\
\hat{\nabla}_{i} \hat{w}_{\hat{\alpha}}^{i}+\frac{1}{4 \mathcal{T}}\left(P^{m}-\mathcal{T} \partial_{\sigma} X^{m}\right)\left(\gamma_{m} \hat{L}\right)_{\hat{\alpha}}-\hat{\chi}_{i} \hat{p}_{\hat{\alpha}}^{i} & =0, \\
\hat{\lambda}^{\hat{\alpha}} \hat{w}_{\hat{\alpha}}^{i}+\epsilon^{i j}\left(\hat{\Sigma}_{\hat{\chi}_{j}}+\partial_{j} \hat{B}\right) & =0, \\
\partial_{i} \hat{\xi}^{\hat{\alpha}}-\hat{\chi}_{i} \hat{\lambda}^{\hat{\alpha}} & =0,
\end{aligned}
$$




$$
\begin{aligned}
\partial_{i} \hat{p}_{\hat{\alpha}}^{i} & =0, \\
\epsilon^{i j} \partial_{i} \hat{A}_{j} & =0 \\
\left(P^{m}-\mathcal{T} \partial_{\sigma} X^{m}\right)\left(\gamma_{m} \hat{\lambda}\right)_{\alpha} & =0 \\
\hat{\lambda}^{\hat{\alpha}} \hat{p}_{\hat{\alpha}}^{i}+\epsilon^{i j} \hat{\nabla}_{j} \hat{\Sigma} & =0 \\
\epsilon^{i j} \hat{\nabla}_{i} \hat{\chi}_{j} & =0 .
\end{aligned}
$$

Due to the constraints (2.14) and (2.19), the action $S_{0}$ is invariant under

$$
\begin{aligned}
& \delta w_{\alpha}^{i}=d_{m}^{i}\left(\gamma^{m} \lambda\right)_{\alpha}+e_{m}^{i}\left(\gamma^{m} \xi\right)_{\alpha}, \quad \delta \hat{w}_{\hat{\alpha}}^{i}=\hat{d}_{m}^{i}\left(\gamma^{m} \hat{\lambda}\right)_{\hat{\alpha}}+\hat{e}_{m}^{i}\left(\gamma^{m} \hat{\xi}\right)_{\hat{\alpha}}, \\
& \delta p_{\alpha}^{i}=e_{m}^{i}\left(\gamma^{m} \lambda\right)_{\alpha}, \quad \delta \hat{p}_{\hat{\alpha}}^{i}=\hat{e}_{m}^{i}\left(\gamma^{m} \hat{\lambda}\right)_{\hat{\alpha}}, \\
& \delta L^{\alpha}=f \lambda^{\alpha}+f_{m n}\left(\gamma^{m n} \lambda\right)^{\alpha}+g \xi^{\alpha}, \quad \delta \hat{L}^{\hat{\alpha}}=\hat{f} \hat{\lambda}^{\hat{\alpha}}+\hat{f}_{m n}\left(\gamma^{m n} \hat{\lambda}\right)^{\hat{\alpha}}+\hat{g} \xi^{\hat{\alpha}},
\end{aligned}
$$

where $d_{m}, e_{m}, f, f_{m n}, g$ (hatted and unhatted) are local parameters. These gauge transformations have a special role in the formalism and will be called pure spinor symmetries. The other gauge symmetries of the model can be summarized by:

1. Worldsheet reparametrization, with parameter $c^{i}=\left\{c^{\tau}, c^{\sigma}\right\}$. Although the transformations of $P_{m}, L^{\alpha}$ and $\hat{L}^{\hat{\alpha}}$ are nontrivial,

$$
\begin{aligned}
& \delta P_{m}=\partial_{\sigma}\left(c^{\sigma} P_{m}\right)+c^{\tau} \partial_{\tau} P_{m}+\frac{1}{4}\left(L_{+} \gamma_{m} \lambda\right)\left(\partial_{\sigma} c^{\tau}\right)-\frac{1}{4}\left(\hat{L}_{-} \gamma_{m} \hat{\lambda}\right)\left(\partial_{\sigma} c^{\tau}\right), \\
& \delta L^{\alpha}=c^{\sigma} \partial_{\sigma} L^{\alpha}+\partial_{\tau}\left(c^{\tau} L^{\alpha}\right)+\partial_{\tau} c^{\sigma}\left(P^{m}+\mathcal{T} \partial_{\sigma} X^{m}\right) \frac{\left(\gamma_{m} \Lambda\right)^{\alpha}}{(\Lambda \lambda)} \\
& \delta \hat{L}^{\hat{\alpha}}=c^{\sigma} \partial_{\sigma} \hat{L}^{\hat{\alpha}}+\partial_{\tau}\left(c^{\tau} \hat{L}^{\hat{\alpha}}\right)-\partial_{\tau} c^{\sigma}\left(P^{m}-\mathcal{T} \partial_{\sigma} X^{m}\right) \frac{\left(\gamma_{m} \hat{\Lambda}\right)^{\hat{\alpha}}}{(\hat{\Lambda} \hat{\lambda})}
\end{aligned}
$$

all the other fields transform covariantly either as worldsheet scalars (e.g. $\delta X^{m}=$ $\left.c^{i} \partial_{i} X^{m}\right)$, vector densities (e.g. $\delta w_{\alpha}^{i}=\partial_{j}\left(c^{j} w_{\alpha}^{i}\right)-w_{\alpha}^{j} \partial_{j} c^{i}$ ) or 1-forms (e.g. $\delta A_{i}=$ $\left.c^{j} \partial_{j} A_{i}+A_{j} \partial_{i} c^{j}\right)$.

2. Particle-like Hamiltonian symmetry, with parameter $a^{ \pm}$. This symmetry is analogous to (2.9) and the transformations can be cast as

$$
\begin{aligned}
\delta X^{m} & =a^{+}\left(P^{m}+\mathcal{T} \partial_{\sigma} X^{m}\right)+a^{-}\left(P^{m}-\mathcal{T} \partial_{\sigma} X^{m}\right), \\
\delta P_{m} & =\mathcal{T} \partial_{\sigma}\left[a^{+}\left(P^{m}+\mathcal{T} \partial_{\sigma} X^{m}\right)\right]-\mathcal{T} \partial_{\sigma}\left[a^{-}\left(P^{m}-\mathcal{T} \partial_{\sigma} X^{m}\right)\right], \\
\delta L^{\alpha} & =2 \mathcal{T} a^{+} \nabla_{\sigma} L^{\alpha}+2 \mathcal{T} \partial_{\tau} a^{+}\left(P^{m}+\mathcal{T} \partial_{\sigma} X^{m}\right) \frac{\left(\gamma_{m} \Lambda\right)^{\alpha}}{(\Lambda \lambda)} \\
\delta w_{\alpha}^{\sigma} & =-\frac{1}{2} a^{+}\left(P^{m}+\mathcal{T} \partial_{\sigma} X^{m}\right)\left(\gamma_{m} L\right)_{\alpha} \\
\delta \hat{L}^{\hat{\alpha}} & =-2 \mathcal{T} a^{-} \hat{\nabla}_{\sigma} \hat{L}^{\hat{\alpha}}+2 \mathcal{T} \partial_{\tau} a^{-}\left(P_{m}-\mathcal{T} \partial_{\sigma} X_{m}\right) \frac{\left(\gamma^{m} \hat{\Lambda}\right)^{\hat{\alpha}}}{(\hat{\lambda} \hat{\Lambda})} \\
\delta \hat{w}_{\hat{\alpha}}^{\sigma} & =\frac{1}{2} a^{-}\left(P^{m}-\mathcal{T} \partial_{\sigma} X^{m}\right)\left(\gamma_{m} \hat{L}\right)_{\hat{\alpha}} .
\end{aligned}
$$

3. Scaling symmetry, with parameters $\{\Omega, \hat{\Omega}\}$. The transformations are

$$
\begin{aligned}
\delta \lambda^{\alpha} & =\Omega \lambda^{\alpha}, & \delta \hat{\lambda}^{\hat{\alpha}} & =\hat{\Omega} \hat{\lambda}^{\hat{\alpha}}, \\
\delta w_{\alpha}^{i} & =-\Omega w_{\alpha}^{i}, & \delta \hat{w}_{\hat{\alpha}}^{i} & =-\Omega \hat{w}_{\hat{\alpha}}^{i}, \\
\delta A_{i} & =-\partial_{i} \Omega, & \delta \hat{A}_{i} & =-\partial_{i} \hat{\Omega}, \\
\delta L^{\alpha} & =-\Omega L^{\alpha}, & \delta \hat{L}^{\hat{\alpha}} & =-\hat{\Omega} \hat{L}^{\hat{\alpha}}, \\
\delta \chi_{i} & =-\Omega \chi_{i}, & \delta \hat{\chi}_{i} & =-\hat{\Omega} \hat{\chi}_{i}, \\
\delta \Sigma & =\Omega \Sigma, & \delta \hat{\Sigma} & =\hat{\Omega} \hat{\Sigma} .
\end{aligned}
$$


4. Fermionic gauge symmetry, with gauge fields $\chi_{i}$ and $\hat{\chi}_{i}$ and parameters $\gamma$ and $\hat{\gamma}$, respectively. The action $S_{0}$ is invariant under the transformations

$$
\begin{aligned}
\delta w_{\alpha}^{i} & =\gamma p_{\alpha}^{i}, & \delta \hat{w}_{\hat{\alpha}}^{i} & =\hat{\gamma} \hat{p}_{\hat{\alpha}}^{i}, \\
\delta B & =\gamma \Sigma, & \delta \hat{B} & =\hat{\gamma} \hat{\Sigma} \\
\delta \xi^{\alpha} & =\gamma \lambda^{\alpha}, & \delta \hat{\xi}^{\hat{\alpha}} & =\hat{\gamma} \hat{\lambda}^{\hat{\alpha}} \\
\delta \chi_{i} & =\nabla_{i} \gamma, & \delta \chi_{i} & =\hat{\nabla}_{i} \hat{\gamma} .
\end{aligned}
$$

5. Curl symmetry, with parameters $\left\{s_{\alpha}, \epsilon_{\alpha}, \hat{s}_{\hat{\alpha}}, \hat{\epsilon}_{\hat{\alpha}}\right\}$. By construction, the reparametrization invariant form of the kinetic terms of the spinors in (2.18) imply the existence of gauge transformations given by

$$
\begin{aligned}
\delta w_{\alpha}^{i} & =\epsilon^{i j}\left(\nabla_{j} s_{\alpha}+\epsilon_{\alpha} \chi_{j}\right), & \delta \hat{w}_{\hat{\alpha}}^{i} & =\epsilon^{i j}\left(\hat{\nabla}_{j} \hat{s}_{\hat{\alpha}}+\hat{\epsilon}_{\hat{\alpha}} \hat{\chi}_{j}\right), \\
\delta B & =-\lambda^{\alpha} s_{\alpha}, & \delta \hat{B} & =-\hat{\lambda}^{\hat{\alpha}} \hat{s}_{\hat{\alpha}}, \\
\delta p_{\alpha}^{i} & =\epsilon^{i j} \partial_{j} \epsilon_{\alpha}, & \delta \hat{p}_{\hat{\alpha}} & =\epsilon^{i j} \partial_{j} \hat{\epsilon}_{\hat{\alpha}}, \\
\delta \Sigma & =-\lambda^{\alpha} \epsilon_{\alpha}, & \delta \hat{\Sigma} & =-\hat{\lambda}^{\hat{\alpha}} \hat{\epsilon}_{\hat{\alpha}} .
\end{aligned}
$$

6. Twistor-like symmetry, with parameters $\theta^{\alpha}$ and $\hat{\theta}^{\hat{\alpha}}$ and gauge transformations

$$
\begin{aligned}
\delta X^{m} & =\frac{1}{4 T}\left[\left(\lambda \gamma^{m} \theta\right)+\left(\hat{\lambda} \gamma^{m} \hat{\theta}\right)\right], & \delta w_{\alpha}^{i}= & -\delta_{\tau}^{i} \frac{1}{4 \mathcal{T}}\left(P_{m}+\mathcal{T} \partial_{\sigma} X^{m}\right)\left(\theta \gamma^{m}\right)_{\alpha} \\
\delta P_{m} & =\frac{1}{4} \nabla_{\sigma}\left[\left(\lambda \gamma^{m} \theta\right)-\left(\hat{\lambda} \gamma^{m} \hat{\theta}\right)\right], & & +\delta_{\sigma}^{i} \frac{1}{8 \mathcal{T}}\left(L \gamma_{m} \lambda\right)\left(\theta \gamma^{m}\right)_{\alpha}, \\
\delta L^{\alpha} & =\nabla_{\tau} \theta^{\alpha}, & \delta \hat{w}_{\hat{\alpha}}^{i}= & -\delta_{\tau}^{i} \frac{1}{4 \mathcal{T}}\left(P_{m}-\mathcal{T} \partial_{\sigma} X^{m}\right)\left(\hat{\theta} \gamma^{m}\right)_{\hat{\alpha}} \\
\delta \hat{L}^{\hat{\alpha}} & =\hat{\nabla}_{\tau} \hat{\theta}^{\hat{\alpha}}, & & -\delta_{\sigma}^{i} \frac{1}{8 \mathcal{T}}\left(\hat{L} \gamma_{m} \hat{\lambda}\right)\left(\hat{\theta} \gamma^{m}\right)_{\hat{\alpha}} .
\end{aligned}
$$

To complete the analysis of the gauge structure of $S_{0}$, consider the gauge-for-gauge transformations with parameters $\phi^{i}$ and $\varphi^{ \pm}$:

$$
\begin{array}{rlrl}
\delta^{\prime} c^{i} & =\phi^{i}, & & \delta^{\prime} \Omega=\phi^{i} A_{i}, \\
\delta^{\prime} a^{ \pm}=\varphi^{ \pm} \mp \phi^{\sigma}, & \delta^{\prime} \hat{\Omega}=\phi^{i} \hat{A}_{i}, \\
\delta^{\prime} s_{\alpha}=\epsilon_{i j} \phi^{j} w_{\alpha}^{i}, & \delta^{\prime} \epsilon_{\alpha}=\epsilon_{i j} \phi^{j} p_{\alpha}^{i} \\
\delta^{\prime} \hat{s}_{\hat{\alpha}}=\epsilon_{i j} \phi^{j} \hat{w}_{\hat{\alpha}}^{i}, & \delta^{\prime} \hat{\epsilon}_{\hat{\alpha}}=\epsilon_{i j} \phi^{j} \hat{p}_{\hat{\alpha}}^{i}, \\
\delta^{\prime} \gamma=-\phi^{i} \chi_{i}, & \delta^{\prime} \theta^{\alpha}=-\phi^{\tau} L^{\alpha}-\varphi^{+}\left(P_{m}+\mathcal{T} \partial_{\sigma} X_{m}\right) \frac{\left(\gamma^{m} \Lambda\right)^{\alpha}}{(\Lambda \lambda)} \\
\delta^{\prime} \hat{\gamma}=-\phi^{i} \hat{\chi}_{i}, & \delta^{\prime} \hat{\theta}^{\hat{\alpha}}=-\phi^{\tau} \hat{L}^{\hat{\alpha}}-\varphi^{-}\left(P_{m}-\mathcal{T} \partial_{\sigma} X_{m}\right) \frac{\left(\gamma^{m} \hat{\Lambda}\right)^{\hat{\alpha}}}{(\hat{\lambda} \hat{\Lambda})}
\end{array}
$$

Up to equations of motion and pure spinor symmetries, cf. equations (2.20) and (2.21), the gauge transformations listed above are left invariant by (2.28):

$$
\begin{aligned}
\delta^{\prime}\left[\delta X^{m}\right]= & \phi^{\tau}\left\{\partial_{\tau} X^{m}-\frac{1}{4 \mathcal{T}}\left(\lambda \gamma^{m} L\right)-\frac{1}{4 \mathcal{T}}\left(\hat{\lambda} \gamma^{m} \hat{L}\right)\right\} \\
& +\frac{1}{4 \mathcal{T}} \varphi^{+}\left\{\left(P_{n}+\mathcal{T} \partial_{\sigma} X_{n}\right)\left(\gamma^{n} \lambda\right)_{\alpha}\right\} \frac{\left(\gamma^{m} \Lambda\right)^{\alpha}}{(\Lambda \lambda)} \\
& +\frac{1}{4 \mathcal{T}} \varphi^{-}\left\{\left(P_{n}-\mathcal{T} \partial_{\sigma} X_{n}\right)\left(\gamma^{n} \hat{\lambda}\right)_{\hat{\alpha}}\right\} \frac{\left(\gamma^{m} \hat{\Lambda}\right)^{\alpha}}{(\hat{\Lambda} \hat{\lambda})}
\end{aligned}
$$




$$
\begin{aligned}
& \delta^{\prime}\left[\delta P_{m}\right]=\phi^{\tau}\left\{\partial_{\tau} P_{m}-\frac{1}{4} \partial_{\sigma}\left(\lambda \gamma_{m} L\right)+\frac{1}{4} \partial_{\sigma}\left(\hat{\lambda} \gamma_{m} \hat{L}\right)\right\} \\
& +\frac{1}{4} \nabla_{\sigma}\left[\varphi^{+}\left\{\left(P_{n}+\mathcal{T} \partial_{\sigma} X_{n}\right)\left(\gamma^{n} \lambda\right)_{\alpha}\right\} \frac{\left(\gamma^{m} \Lambda\right)^{\alpha}}{(\Lambda \lambda)}\right] \\
& -\frac{1}{4} \hat{\nabla}_{\sigma}\left[\varphi^{-}\left\{\left(P_{n}-\mathcal{T} \partial_{\sigma} X_{n}\right)\left(\gamma^{n} \hat{\lambda}\right)_{\hat{\alpha}}\right\} \frac{\left(\gamma^{m} \hat{\Lambda}\right)^{\hat{\alpha}}}{(\hat{\Lambda} \hat{\lambda})}\right], \\
& \delta^{\prime}\left[\delta \lambda^{\alpha}\right]=\phi^{i}\left\{\nabla_{i} \lambda^{\alpha}\right\} \text {, } \\
& \delta^{\prime}\left[\delta w_{\alpha}^{i}\right]=\phi^{i}\left\{\nabla_{j} w_{\alpha}^{j}+\frac{1}{4 T}\left(P_{m}+\mathcal{T} \partial_{\sigma} X_{m}\right)\left(\gamma^{m} L\right)_{\alpha}-\chi_{j} p_{\alpha}^{j}\right\} \\
& +\frac{1}{16 \mathcal{T}} \delta_{\sigma}^{i} \phi^{\tau}\left(L \gamma_{m} L\right)\left(\gamma^{m} \lambda\right)_{\alpha}+\frac{1}{8 \mathcal{T}} \delta_{\sigma}^{i} \varphi^{+}\left(P_{n}+\mathcal{T} \partial_{\sigma} X_{n}\right)\left(L \gamma^{m} \gamma^{n} \Lambda\right) \frac{\left(\gamma^{m} \lambda\right)_{\alpha}}{(\Lambda \lambda)} \\
& +\frac{1}{4 \mathcal{T}} \delta_{\tau}^{i} \varphi^{+}\left\{\left(P_{m}+\mathcal{T} \partial_{\sigma} X_{m}\right)\left(P^{m}+\mathcal{T} \partial_{\sigma} X^{m}\right)\right\} \frac{\Lambda_{\alpha}}{(\Lambda \lambda)}, \\
& -\frac{1}{8 \mathcal{T}} \delta_{\sigma}^{i} \varphi^{+}\left\{\left(P_{n}+\mathcal{T} \partial_{\sigma} X_{n}\right)\left(\gamma^{n} \lambda\right)_{\beta}\right\}\left(\gamma_{m} L\right)_{\alpha} \frac{\left(\gamma^{m} \Lambda\right)^{\beta}}{(\Lambda \lambda)}, \\
& \delta^{\prime}\left[\delta A_{i}\right]=\phi^{j}\left\{\partial_{j} A_{i}-\partial_{i} A_{j}\right\} \\
& \delta^{\prime}[\delta B]=\phi^{k} \epsilon_{k i}\left\{\lambda^{\alpha} w_{\alpha}^{i}+\epsilon^{i j}\left(\Sigma \chi_{j}+\partial_{j} B\right)\right\}, \\
& \delta^{\prime}\left[\delta L^{\alpha}\right]=\varphi^{+}\left(P_{m}+\mathcal{T} \partial_{\sigma} X_{m}\right)\left\{\nabla_{\tau} \lambda^{\beta}\right\} \Lambda_{\beta} \frac{\left(\gamma^{m} \Lambda\right)^{\alpha}}{(\Lambda \lambda)^{2}}-\frac{1}{2} \varphi^{+}\left(L \gamma_{m}\right)_{\beta}\left\{\nabla_{\sigma} \lambda^{\beta}\right\} \frac{\left(\gamma^{m} \Lambda\right)^{\alpha}}{(\Lambda \lambda)} \\
& -\varphi^{+}\left\{\partial_{\tau} P_{m}-\frac{1}{4} \partial_{\sigma}\left(L \gamma_{m} \lambda\right)+\frac{1}{4} \partial_{\sigma}\left(\hat{L} \gamma_{m} \hat{\lambda}\right)\right\} \frac{\left(\gamma^{m} \Lambda\right)^{\alpha}}{(\Lambda \lambda)} \\
& -\mathcal{T} \varphi^{+} \partial_{\sigma}\left\{\partial_{\tau} X_{m}-\frac{1}{4 \mathcal{T}}\left(L \gamma^{m} \lambda\right)-\frac{1}{4 \mathcal{T}}\left(\hat{L} \gamma^{m} \hat{\lambda}\right)\right\} \frac{\left(\gamma^{m} \Lambda\right)^{\alpha}}{(\Lambda \lambda)} \\
& -\frac{1}{8} \varphi^{+}\left(\Lambda \gamma_{m n} \nabla_{\sigma} L\right) \frac{\left(\gamma^{m n} \lambda\right)^{\alpha}}{(\Lambda \lambda)}-\frac{1}{4} \varphi^{+}\left(\Lambda \nabla_{\sigma} L\right) \frac{\lambda^{\alpha}}{(\Lambda \lambda)}, \\
& \delta^{\prime}\left[\delta \xi^{\alpha}\right]=\phi^{i}\left\{\partial_{i} \xi^{\alpha}-\chi_{i} \lambda^{\alpha}\right\}, \\
& \delta^{\prime}\left[\delta p_{\alpha}^{i}\right]=\phi^{i}\left\{\partial_{j} p_{\alpha}^{j}\right\} \\
& \delta^{\prime}\left[\delta \chi_{i}\right]=\phi^{j}\left\{\nabla_{j} \chi_{i}-\nabla_{i} \chi_{j}\right\}, \\
& \delta^{\prime}[\delta \Sigma]=\phi^{k} \epsilon_{k j}\left\{\lambda^{\alpha} p_{\alpha}^{i}+\epsilon^{i j} \nabla_{j} \Sigma\right\} .
\end{aligned}
$$

Similar equations hold for the hatted sector. This confirms that worldsheet reparametrization is a redundant symmetry of the action, since the parameters $\phi^{i}$ and $\varphi^{ \pm}$can be used to set $c^{i}=a^{ \pm}=0$. Furthermore, their gauge-for-gauge transformations in (2.28) involve only simple field shifts, i.e. no derivatives of the gauge-for-gauge parameters, therefore generating no dynamical ghost-for-ghosts. Consequently, the gauge symmetries parametrized by $c^{i}$ and $a^{ \pm}$can be disregarded in the construction of the master action within the BatalinVilkovisky formalism. It will be demonstrated next that the quantization of the action $S_{0}$ leads to the pure spinor superstring.

\subsection{The pure spinor master action}

In order to build the pure spinor master action in the Batalin-Vilkovisky formalism, the gauge parameters discussed above will be promoted to dynamical variables. The field 
content of the model will be collectively denoted by $\Phi^{I}$, with the index $I$ running over the set

$$
\begin{aligned}
\Phi^{I}= & \left\{X^{m}, P_{m}, w_{\alpha}^{i}, \lambda^{\alpha}, L^{\alpha}, A_{i}, B, p_{\alpha}^{i}, \xi^{\alpha}, \chi_{i}, \Sigma, \Omega, \theta^{\alpha}, s_{\alpha}, \epsilon_{\alpha}, \gamma\right. \\
& \left.\hat{w}_{\hat{\alpha}}^{i}, \hat{\lambda}^{\hat{\alpha}}, \hat{L}^{\hat{\alpha}}, \hat{A}_{i}, \hat{B}, \hat{p}_{\hat{\alpha}}^{i}, \hat{\xi}^{\hat{\alpha}}, \hat{\chi}_{i}, \hat{\Sigma}, \hat{\Omega}, \hat{\theta}^{\hat{\alpha}}, \hat{s}_{\hat{\alpha}}, \hat{\epsilon}_{\hat{\alpha}}, \hat{\gamma}\right\} .
\end{aligned}
$$

As usual, ghost fields and the correspondent gauge parameters have opposite statistics, therefore $\left\{\Omega, \hat{\Omega}, \theta^{\alpha}, \hat{\theta}^{\hat{\alpha}}, s_{\alpha}, \hat{s}_{\hat{\alpha}}\right\}$ are Grassmann odd while $\left\{\epsilon_{\alpha}, \hat{\epsilon}_{\hat{\alpha}}, \gamma, \hat{\gamma}\right\}$ are Grassmann even fields. Following the discussion at the end of the previous subsection, the gauge parameters $c^{i}$ and $a^{ \pm}$, and the gauge-for-gauge parameters $\phi^{i}$ and $\varphi^{ \pm}$will be ignored.

For every field $\Phi^{I}$ there is an antifield $\Phi_{I}^{*}$ associated, with opposite statistics, and the antifield set is given by

$$
\begin{aligned}
\Phi_{I}^{*}= & \left\{X_{m}^{*}, P_{*}^{m}, w_{i *}^{\alpha}, \lambda_{\alpha}^{*}, L_{\alpha}^{*}, A_{*}^{i}, B^{*}, p_{i *}^{\alpha}, \xi_{\alpha}^{*}, \chi_{*}^{i}, \Sigma^{*}, \Omega^{*}, \theta_{\alpha}^{*}, s_{*}^{\alpha}, \epsilon_{*}^{\alpha}, \gamma^{*},\right. \\
& \left.\hat{w}_{i *}^{\hat{\alpha}}, \hat{\lambda}_{\hat{\alpha}}^{*}, \hat{L}_{\hat{\alpha}}^{*}, \hat{A}_{*}^{i}, \hat{B}^{*}, \hat{p}_{i *}^{\hat{\alpha}}, \hat{\xi}_{\hat{\alpha}}^{*}, \hat{\chi}_{*}^{i}, \hat{\Sigma}^{*}, \hat{\Omega}^{*}, \hat{\theta}_{\hat{\alpha}}^{*}, \hat{s}_{*}^{\hat{\alpha}}, \hat{\epsilon}_{*}^{\hat{\alpha}}, \hat{\gamma}^{*}\right\} .
\end{aligned}
$$

By definition, fields and antifields are conjugate to each other, satisfying the antibracket relation

$$
\left\{\Phi_{I}^{*}, \Phi^{J}\right\}=\delta_{I}^{J} .
$$

In general, the antibrackets between two operators $\mathcal{O}_{1}$ and $\mathcal{O}_{2}$ are defined as

$$
\left\{\mathcal{O}_{1}, \mathcal{O}_{2}\right\} \equiv \sum_{I}\left\{\mathcal{O}_{1}\left(\frac{\overleftarrow{\partial}}{\partial \Phi_{I}^{*}} \frac{\partial}{\partial \Phi^{I}}-\frac{\overleftarrow{\partial}}{\partial \Phi^{I}} \frac{\partial}{\partial \Phi_{I}^{*}}\right) \mathcal{O}_{2}\right\}
$$

from which equation (2.32) follows. However, as consequence of the constraints (2.14) and (2.19), not all the components of $\Phi^{I}$ and $\Phi_{I}^{*}$ are independent. In fact, the pure spinor constraints are generalized to

$$
\begin{aligned}
\left(\lambda \gamma^{m} \lambda\right) & =0, & \left(\lambda \gamma^{m} p_{i *}\right)+\left(\xi \gamma^{m} w_{i *}\right) & =0, \\
\left(\lambda \gamma^{m} \xi\right) & =0, & \lambda^{\alpha} \theta_{\alpha}^{*}+w_{\tau *}^{\alpha} L_{\alpha}^{*} & =0, \\
\left(\lambda \gamma^{m} w_{i *}\right) & =0, & \xi^{\alpha} \theta_{\alpha}^{*}+p_{\tau *}^{\alpha} L_{\alpha}^{*} & =0, \\
\lambda^{\alpha} L_{\alpha}^{*} & =0, & \left(\lambda \gamma^{m n} \theta^{*}\right)+\left(w_{\tau *} \gamma^{m n} L^{*}\right) & =0, \\
\left(\lambda \gamma^{m n} L^{*}\right) & =0, & \left(\lambda \gamma^{m} s_{*}\right)-\frac{1}{2} \epsilon^{i j}\left(w_{i *} \gamma^{m} w_{j *}\right) & =0, \\
\xi^{\alpha} L_{\alpha}^{*} & =0, & \left(\lambda \gamma^{m} \epsilon_{*}\right)+\left(\xi \gamma^{m} s_{*}\right)-\epsilon^{i j}\left(p_{i *} \gamma^{m} w_{j *}\right) & =0,
\end{aligned}
$$

and analogous constraints on the hatted sector, and the antibracket (2.32) cannot be naively computed. For example,

$$
\left\{\lambda_{\alpha}^{*},\left(\lambda \gamma^{m} \lambda\right)\right\}=2\left(\gamma^{m} \lambda\right)_{\alpha},
$$

which is not compatible with the constraint $\left(\lambda \gamma^{m} \lambda\right)=0$. This contradiction arises because there is an intrinsic gauge freedom implied by the constraints (2.34), which only have vanishing antibrackets with operators invariant under the pure spinor gauge transformations given by

$$
\begin{aligned}
\delta \lambda_{\alpha}^{*}= & b_{m}\left(\gamma^{m} \lambda\right)_{\alpha}+c_{m}\left(\gamma^{m} \xi\right)_{\alpha}-d_{m}^{i}\left(\gamma^{m} w_{i *}\right)_{\alpha}-e_{m}^{i}\left(\gamma^{m} p_{i *}\right)_{\alpha}-f L_{\alpha}^{*} \\
& +f_{m n}\left(\gamma^{m n} L^{*}\right)_{\alpha}-\bar{f} \theta_{\alpha}^{*}+\bar{f}_{m n}\left(\gamma^{m n} \theta^{*}\right)_{\alpha}-h_{m}\left(\gamma^{m} s_{*}\right)_{\alpha}-\bar{h}_{m}\left(\gamma^{m} \epsilon_{*}\right)_{\alpha},
\end{aligned}
$$




$$
\begin{aligned}
\delta \xi_{\alpha}^{*}= & c_{m}\left(\gamma^{m} \lambda\right)_{\alpha}+e_{m}^{i}\left(\gamma^{m} w_{i *}\right)_{\alpha}+g L_{\alpha}^{*}-\bar{g} \theta_{\alpha}^{*}-\bar{h}_{m}\left(\gamma^{m} s_{*}\right)_{\alpha} \\
\delta w_{\alpha}^{i}= & d_{m}^{i}\left(\gamma^{m} \lambda\right)_{\alpha}+e_{m}^{i}\left(\gamma^{m} \xi\right)_{\alpha}-\delta_{\tau}^{i} \bar{f} L_{\alpha}^{*}+\delta_{\tau}^{i} \bar{f}_{m n}\left(\gamma^{m n} L^{*}\right)_{\alpha}+\epsilon^{i j} h_{m}\left(\gamma^{m} w_{j *}\right)_{\alpha} \\
& +\epsilon^{i j} \bar{h}_{m}\left(\gamma^{m} p_{j *}\right)_{\alpha} \\
\delta p_{\alpha}^{i}= & e_{m}^{i}\left(\gamma^{m} \lambda\right)_{\alpha}+\delta_{\tau}^{i} \bar{g} L_{\alpha}^{*}-\epsilon^{i j} \bar{h}_{m}\left(\gamma^{m} w_{j *}\right)_{\alpha} \\
\delta L^{\alpha}= & f \lambda^{\alpha}+f_{m n}\left(\gamma^{m n} \lambda\right)^{\alpha}+g \xi^{\alpha}+\bar{f} w_{\tau *}^{\alpha}+\bar{f}_{m n}\left(\gamma^{m n} w_{\tau *}\right)^{\alpha}+\bar{g} p_{\tau *}^{\alpha} \\
\delta \theta^{\alpha}= & \bar{f} \lambda^{\alpha}+\bar{f}_{m n}\left(\gamma^{m n} \lambda\right)^{\alpha}+\bar{g} \xi^{\alpha} \\
\delta s_{\alpha}= & h_{m}\left(\gamma^{m} \lambda\right)_{\alpha}+\bar{h}_{m}\left(\gamma^{m} \xi\right)_{\alpha} \\
\delta \epsilon_{\alpha}= & \bar{h}_{m}\left(\gamma^{m} \lambda\right)_{\alpha}
\end{aligned}
$$

where $b_{m}, c_{m}, d_{m}, e_{m}, f, f_{m n}, g, h_{m}, \bar{f}, \bar{f}_{m n}, \bar{g}$ and $\bar{h}_{m}$ are local parameters. Again, similar transformations exist for the hatted sector.

The pure spinor master action has to be concomitantly determined with the pure spinor constraints (2.34) and symmetries (2.36). It can be cast as

$$
S=S_{0}+S_{1}+S_{2}+S_{3}
$$

where $S_{0}$ is displayed in (2.18) and

$$
\begin{aligned}
& S_{1}=\int d \tau d \sigma\left\{\frac{1}{4 \mathcal{T}}\left(\lambda \gamma^{m} \theta\right) X_{m}^{*}-\frac{1}{4}\left(\lambda \gamma_{m} \theta\right) \partial_{\sigma} P_{*}^{m}-\frac{1}{4 \mathcal{T}}\left(P_{m}+T \partial_{\sigma} X_{m}\right)\left(\theta \gamma^{m} w_{\tau *}\right)\right\} \\
& +\int d \tau d \sigma\left\{\frac{1}{8 \mathcal{T}}\left(L \gamma_{m} \lambda\right)\left(\theta \gamma^{m} w_{\sigma *}\right)+\left(\nabla_{\tau} \theta^{\alpha}\right) L_{\alpha}^{*}+\epsilon^{i j}\left(\nabla_{j} s_{\alpha}\right) w_{i *}^{\alpha}-\lambda^{\alpha} s_{\alpha} B^{*}\right\} \\
& +\int d \tau d \sigma\left\{\Omega \lambda^{\alpha} \lambda_{\alpha}^{*}-\Omega w_{\alpha}^{i} w_{i *}^{\alpha}-\left(\partial_{i} \Omega\right) A_{*}^{i}-\Omega L^{\alpha} L_{\alpha}^{*}-\Omega \chi_{i} \chi_{*}^{i}+\Omega \Sigma \Sigma^{*}\right\} \\
& +\int d \tau d \sigma\left\{\gamma p_{\alpha}^{i} w_{i *}^{\alpha}+\gamma \Sigma B^{*}+\gamma \lambda^{\alpha} \xi_{\alpha}^{*}+\left(\nabla_{i} \gamma\right) \chi_{*}^{i}\right\} \\
& +\int d \tau d \sigma\left\{\epsilon^{i j} \epsilon_{\alpha} \chi_{j} w_{i *}^{\alpha}+\epsilon^{i j}\left(\partial_{j} \epsilon_{\alpha}\right) p_{i *}^{\alpha}-\lambda^{\alpha} \epsilon_{\alpha} \Sigma^{*}\right\} \\
& +\int d \tau d \sigma\left\{\frac{1}{4 \mathcal{T}}\left(\hat{\lambda} \gamma^{m} \hat{\theta}\right) X_{m}^{*}+\frac{1}{4}\left(\hat{\lambda} \gamma_{m} \hat{\theta}\right) \partial_{\sigma} P_{*}^{m}-\frac{1}{4 \mathcal{T}}\left(P_{m}-\mathcal{T} \partial_{\sigma} X_{m}\right)\left(\hat{\theta} \gamma^{m} \hat{w}_{\tau *}\right)\right\} \\
& +\int d \tau d \sigma\left\{-\frac{1}{8 \mathcal{T}}\left(\hat{L} \gamma_{m} \hat{\lambda}\right)\left(\hat{\theta} \gamma^{m} \hat{w}_{\sigma *}\right)+\left(\hat{\nabla}_{\tau} \hat{\theta}^{\hat{\alpha}}\right) \hat{L}_{\hat{\alpha}}^{*}+\epsilon^{i j}\left(\hat{\nabla}_{j} \hat{s}_{\hat{\alpha}}\right) \hat{w}_{i *}^{\hat{\alpha}}-\hat{\lambda}^{\hat{\alpha}} \hat{s}_{\hat{\alpha}} \hat{B}^{*}\right\} \\
& +\int d \tau d \sigma\left\{\hat{\Omega} \hat{\lambda}^{\hat{\alpha}} \hat{\lambda}_{\hat{\alpha}}^{*}-\hat{\Omega} \hat{w}_{\hat{\alpha}}^{i} \hat{w}_{i *}^{\hat{\alpha}}-\left(\partial_{i} \hat{\Omega}\right) \hat{A}_{*}^{i}-\hat{\Omega} \hat{L}^{\hat{\alpha}} \hat{L}_{\hat{\alpha}}^{*}-\hat{\Omega} \hat{\chi}_{i} \hat{\chi}_{*}^{i}+\hat{\Omega} \hat{\Sigma} \hat{\Sigma}^{*}\right\} \\
& +\int d \tau d \sigma\left\{\hat{\gamma} \hat{p}_{\hat{\alpha}}^{i} \hat{w}_{i *}^{\hat{\alpha}}+\hat{\gamma} \hat{\Sigma} \hat{B}^{*}+\hat{\gamma} \hat{\lambda}^{\hat{\alpha}} \hat{\xi}_{\hat{\alpha}}^{*}+\left(\hat{\nabla}_{i} \hat{\gamma}\right) \hat{\chi}_{*}^{i}\right\} \\
& +\int d \tau d \sigma\left\{\epsilon^{i j} \hat{\epsilon}_{\hat{\alpha}} \hat{\chi}_{j} \hat{w}_{i *}^{\hat{\alpha}}+\epsilon^{i j}\left(\partial_{j} \hat{\epsilon}_{\hat{\alpha}}\right) \hat{p}_{i *}^{\hat{\alpha}}-\hat{\lambda}^{\hat{\alpha}} \hat{\epsilon}_{\hat{\alpha}} \hat{\Sigma}^{*}\right\}, \\
& S_{2}=\int d \tau d \sigma\left\{-\Omega \theta^{\alpha} \theta_{\alpha}^{*}-\Omega s_{\alpha} s_{*}^{\alpha}-\Omega \gamma \gamma^{*}-\gamma \epsilon_{\alpha} s_{*}^{\alpha}\right\} \\
& +\int d \tau d \sigma\left\{-\hat{\Omega} \hat{\theta}^{\hat{\alpha}} \hat{\theta}_{\hat{\alpha}}^{*}-\hat{\Omega} \hat{s}_{\hat{\alpha}} \hat{s}_{*}^{\hat{\alpha}}-\hat{\Omega} \hat{\gamma} \hat{\gamma}^{*}-\hat{\gamma} \hat{\epsilon}_{\hat{\alpha}} \hat{s}_{*}^{\hat{\alpha}}\right\},
\end{aligned}
$$




$$
\begin{aligned}
S_{3}= & \int d \tau d \sigma\left\{\frac{1}{16 \mathcal{T}}\left(\theta \gamma^{m} s_{*}\right)\left(\lambda \gamma_{m} \theta\right)+\frac{1}{16 \mathcal{T}}\left(w_{\tau *} \gamma^{m} \theta\right)\left(w_{\sigma *} \gamma_{m} \theta\right)\right\} \\
& -\int d \tau d \sigma\left\{\frac{1}{16 \mathcal{T}}\left(\hat{\theta} \gamma^{m} \hat{s}_{*}\right)\left(\hat{\lambda} \gamma_{m} \hat{\theta}\right)+\frac{1}{16 \mathcal{T}}\left(\hat{w}_{\tau *} \gamma^{m} \hat{\theta}\right)\left(\hat{w}_{\sigma *} \gamma_{m} \hat{\theta}\right)\right\} .
\end{aligned}
$$

$S_{1}$ is connected to the gauge transformations of the action $S_{0}$, while $S_{2}$ represents the extension of the gauge algebra to the ghost fields. The last piece, $S_{3}$, is required in order for $S$ to satisfy the master equation

$$
\{S, S\}=0 .
$$

By construction, the master action is invariant under the BV transformations defined as

$$
\delta_{\mathrm{BV}} \mathcal{O} \equiv\{S, \mathcal{O}\},
$$

for any operator $\mathcal{O}$. Naturally, the BV transformations of the fundamental fields in the action $S_{0}$ have a similar structure to their gauge transformations and are given by

$$
\begin{aligned}
& \delta_{\mathrm{BV}} X^{m}=\frac{1}{4 \mathcal{T}}\left(\lambda \gamma^{m} \theta\right)+\frac{1}{4 \mathcal{T}}\left(\hat{\lambda} \gamma^{m} \hat{\theta}\right), \\
& \delta_{\mathrm{BV}} P_{m}=\frac{1}{4} \nabla_{\sigma}\left(\lambda \gamma^{m} \theta\right)-\frac{1}{4} \hat{\nabla}_{\sigma}\left(\hat{\lambda} \gamma^{m} \hat{\theta}\right), \\
& \delta_{\mathrm{BV}} \lambda^{\alpha}=\Omega \lambda^{\alpha}, \\
& \delta_{\mathrm{BV}} w_{\alpha}^{i}=-\Omega w_{\alpha}^{i}+\epsilon^{i j}\left(\nabla_{j} s_{\alpha}+\epsilon_{\alpha} \chi_{j}\right)+\delta_{\sigma}^{i} \frac{1}{8 \mathcal{T}}\left[\left(L_{+} \gamma_{m} \lambda\right)-\frac{1}{2}\left(w_{\tau *} \gamma_{m} \theta\right)\right]\left(\gamma^{m} \theta\right)_{\alpha} \\
& +\gamma p_{\alpha}^{i}-\delta_{\tau}^{i} \frac{1}{4 \mathcal{T}}\left[P_{m}+\mathcal{T} \partial_{\sigma} X_{m}+\frac{1}{4}\left(w_{\sigma *} \gamma_{m} \theta\right)\right]\left(\gamma^{m} \theta\right)_{\alpha}, \\
& \delta_{\mathrm{BV}} A_{i}=-\partial_{i} \Omega, \\
& \delta_{\mathrm{BV}} B=\gamma \Sigma-\lambda^{\alpha} s_{\alpha}, \\
& \delta_{\mathrm{BV}} L^{\alpha}=\nabla_{\tau} \theta^{\alpha}-\Omega L^{\alpha} \text {, } \\
& \delta_{\mathrm{BV}} \xi^{\alpha}=\gamma \lambda^{\alpha} \\
& \delta_{\mathrm{BV}} p_{\alpha}^{i}=\epsilon^{i j} \partial_{j} \epsilon_{\alpha}, \\
& \delta_{\mathrm{BV}} \chi_{i}=\nabla_{i} \gamma-\Omega \chi_{i}, \\
& \delta_{\mathrm{BV}} \Sigma=\Omega \Sigma-\lambda^{\alpha} \epsilon_{\alpha}, \\
& \delta_{\mathrm{BV}} \hat{\lambda}^{\hat{\alpha}}=\hat{\Omega} \hat{\lambda}^{\hat{\alpha}} \\
& \delta_{\mathrm{BV}} \hat{w}_{\hat{\alpha}}^{i}=-\Omega \hat{w}_{\hat{\alpha}}^{i}+\epsilon^{i j}\left(\hat{\nabla}_{j} \hat{s}_{\alpha}+\hat{\epsilon}_{\alpha} \hat{\chi}_{j}\right)-\delta_{\sigma}^{i} \frac{1}{8 \mathcal{T}}\left[\left(\hat{L} \gamma_{m} \hat{\lambda}\right)-\frac{1}{2}\left(\hat{w}_{\tau *} \gamma_{m} \hat{\theta}\right)\right]\left(\gamma^{m} \hat{\theta}\right)_{\hat{\alpha}} \\
& +\hat{\gamma} \hat{p}_{\hat{\alpha}}^{i}-\delta_{\tau}^{i} \frac{1}{4 \mathcal{T}}\left[P_{m}-\mathcal{T} \partial_{\sigma} X_{m}-\frac{1}{4}\left(\hat{w}_{\sigma *} \gamma_{m} \hat{\theta}\right)\right]\left(\gamma^{m} \hat{\theta}\right)_{\hat{\alpha}}, \\
& \delta_{\mathrm{BV}} \hat{A}_{i}=-\partial_{i} \hat{\Omega} \text {, } \\
& \delta_{\mathrm{BV}} \hat{B}=\hat{\gamma} \hat{\Sigma}-\hat{\lambda}^{\hat{\alpha}} \hat{s}_{\hat{\alpha}}, \\
& \delta_{\mathrm{BV}} \hat{L}^{\hat{\alpha}}=\hat{\nabla}_{\tau} \hat{\theta}^{\hat{\alpha}}-\hat{\Omega} \hat{L}^{\hat{\alpha}}, \\
& \delta_{\mathrm{BV}} \hat{\xi}^{\hat{\alpha}}=\hat{\gamma} \hat{\lambda}^{\hat{\alpha}}, \\
& \delta_{\mathrm{Bv}} \hat{p}_{\hat{\alpha}}^{i}=\epsilon^{i j} \partial_{j} \hat{\epsilon}_{\hat{\alpha}},
\end{aligned}
$$




$$
\begin{aligned}
\delta_{\mathrm{BV}} \hat{\chi}_{i} & =\hat{\nabla}_{i} \hat{\gamma}-\hat{\Omega} \hat{\chi}_{i}, \\
\delta_{\mathrm{BV}} \hat{\Sigma} & =\hat{\Omega} \hat{\Sigma}-\hat{\lambda}^{\hat{\alpha}_{\hat{\epsilon}}} \hat{\epsilon}_{\hat{\alpha}}
\end{aligned}
$$

In general, the BV transformations are nilpotent. However, due to the pure spinor constraints (2.34), the transformations above are nilpotent up to pure spinor gauge transformations. For example,

$$
\begin{aligned}
\delta_{\mathrm{BV}}^{2} w_{\alpha}^{i} & =\frac{1}{16 \mathcal{T}} \epsilon^{i j}\left(\theta \gamma_{m} \nabla_{j} \theta\right)\left(\gamma^{m} \lambda\right)_{\alpha} \\
\delta_{\mathrm{BV}}^{2} \hat{w}_{\hat{\alpha}}^{i} & =-\frac{1}{16 \mathcal{T}} \epsilon^{i j}\left(\hat{\theta} \gamma^{m} \hat{\nabla}_{j} \hat{\theta}\right)\left(\gamma^{m} \hat{\lambda}\right)_{\hat{\alpha}}
\end{aligned}
$$

With the promotion of gauge parameters to ghost fields, their BV transformations are nontrivial and can be cast as

$$
\begin{aligned}
\delta_{\mathrm{BV}} \theta^{\alpha} & =-\Omega \theta^{\alpha}, \\
\delta_{\mathrm{BV}} s_{\alpha} & =-\Omega s_{\alpha}-\gamma \epsilon_{\alpha}-\frac{1}{16 \mathcal{T}}\left(\lambda \gamma_{m} \theta\right)\left(\gamma^{m} \theta\right)_{\alpha}, \\
\delta_{\mathrm{BV}} \Omega & =0, \\
\delta_{\mathrm{BV}} \gamma & =-\Omega \gamma, \\
\delta_{\mathrm{BV}} \epsilon_{\alpha} & =0, \\
\delta_{\mathrm{BV}} \hat{\theta}^{\hat{\alpha}} & =-\hat{\Omega} \hat{\theta}^{\hat{\alpha}}, \\
\delta_{\mathrm{BV}} \hat{s}_{\hat{\alpha}} & =-\hat{\Omega} \hat{s}_{\hat{\alpha}}-\hat{\gamma} \hat{\epsilon}_{\hat{\alpha}}+\frac{1}{16 \mathcal{T}}\left(\hat{\lambda} \gamma_{m} \hat{\theta}\right)\left(\gamma^{m} \hat{\theta}\right)_{\hat{\alpha}}, \\
\delta_{\mathrm{BV}} \hat{\Omega} & =0, \\
\delta_{\mathrm{BV}} \hat{\gamma} & =-\hat{\Omega} \hat{\gamma}, \\
\delta_{\mathrm{BV}} \hat{\epsilon}_{\hat{\alpha}} & =0 .
\end{aligned}
$$

For completeness, the BV transformations for the antifields are given by

$$
\begin{aligned}
\delta_{\mathrm{BV}} X_{m}^{*}= & \partial_{\tau} P_{m}-\frac{1}{4} \partial_{\sigma}\left[\left(L \gamma_{m} \lambda\right)+\left(\theta \gamma_{m} w_{\tau *}\right)-\left(\hat{L} \gamma_{m} \hat{\lambda}\right)-\left(\hat{\theta} \gamma_{m} \hat{w}_{\tau *}\right)\right] \\
\delta_{\mathrm{BV}} P_{*}^{m}= & -\partial_{\tau} X^{m}+\frac{1}{4 \mathcal{T}}\left[\left(L \gamma^{m} \lambda\right)+\left(\theta \gamma^{m} w_{\tau *}\right)+\left(\hat{L} \gamma^{m} \hat{\lambda}\right)+\left(\hat{\theta} \gamma^{m} \hat{w}_{\tau *}\right)\right] \\
\delta_{\mathrm{BV}} \lambda_{\alpha}^{*}= & \nabla_{i} w_{\alpha}^{i}-\chi_{i} p_{\alpha}^{i}+s_{\alpha} B^{*}+\frac{1}{4 \mathcal{T}}\left[P^{m}+\mathcal{T} \partial_{\sigma} X^{m}-\frac{1}{2}\left(\theta \gamma^{m} w_{\sigma *}\right)\right]\left(\gamma_{m} L\right)_{\alpha} \\
& -\Omega \lambda_{\alpha}^{*}-\gamma \xi_{\alpha}^{*}+\epsilon_{\alpha} \Sigma^{*}+\frac{1}{4 \mathcal{T}}\left[\eta^{m n} X_{n}^{*}-\mathcal{T} \partial_{\sigma} P_{*}^{m}-\frac{1}{4}\left(\theta \gamma^{m} s_{*}\right)\right]\left(\gamma_{m} \theta\right)_{\alpha}, \\
\delta_{\mathrm{BV}} w_{i *}^{\alpha}= & -\nabla_{i} \lambda^{\alpha}+\Omega w_{i *}^{\alpha} \\
\delta_{\mathrm{BV}} A_{*}^{i}= & -\lambda^{\alpha} w_{\alpha}^{i}-\epsilon^{i j} \partial_{j} B-\Sigma \epsilon^{i j} \chi_{j}-\epsilon^{i j} s_{\alpha} w_{j *}^{\alpha}+\delta_{\tau}^{i} \theta^{\alpha} L_{\alpha}^{*}+\gamma \chi_{*}^{i}, \\
\delta_{\mathrm{BV}} B^{*}= & -\epsilon^{i j} \partial_{i} A_{j}, \\
\delta_{\mathrm{BV}} L_{\alpha}^{*}= & \frac{1}{4 \mathcal{T}}\left[P^{m}+\mathcal{T} \partial_{\sigma} X^{m}-\frac{1}{2}\left(\theta \gamma^{m} w_{\sigma *}\right)\right]\left(\gamma_{m} \lambda\right)_{\alpha}+\Omega L_{\alpha}^{*} \\
\delta_{\mathrm{BV}} \xi_{\alpha}^{*}= & \partial_{i} p_{\alpha}^{i}
\end{aligned}
$$




$$
\begin{aligned}
\delta_{\mathrm{BV}} p_{i *}^{\alpha}= & \partial_{i} \xi^{\alpha}-\lambda^{\alpha} \chi_{i}+\gamma w_{i *}^{\alpha}, \\
\delta_{\mathrm{BV}} \chi_{*}^{i}= & \lambda^{\alpha} p_{\alpha}^{i}+\epsilon^{i j} \nabla_{j} \Sigma+\Omega \chi_{*}^{i}-\epsilon^{i j} \epsilon_{\alpha} w_{j *}^{\alpha}, \\
\delta_{\mathrm{BV}} \Sigma^{*}= & -\epsilon^{i j} \nabla_{i} \chi_{j}-\Omega \Sigma^{*}+\gamma B^{*}, \\
\delta_{\mathrm{BV}} \hat{\lambda}_{\hat{\alpha}}^{*}= & \hat{\nabla}_{i} \hat{w}_{\hat{\alpha}}^{i}-\hat{\chi}_{i} \hat{p}_{\hat{\alpha}}^{i}+\hat{s}_{\hat{\alpha}} \hat{B}^{*}+\frac{1}{4 \mathcal{T}}\left[P^{m}-\mathcal{T} \partial_{\sigma} X^{m}+\frac{1}{2}\left(\hat{\theta} \gamma^{m} \hat{w}_{\sigma *}\right)\right]\left(\gamma_{m} \hat{L}\right)_{\hat{\alpha}} \\
& -\hat{\Omega} \hat{\lambda}_{\hat{\alpha}}^{*}-\hat{\gamma} \hat{\xi}_{\hat{\alpha}}^{*}+\hat{\epsilon}_{\hat{\alpha}} \hat{\Sigma}^{*}+\frac{1}{4 \mathcal{T}}\left[\eta^{m n} X_{n}^{*}+\mathcal{T} \partial_{\sigma} P_{*}^{m}+\frac{1}{4}\left(\hat{\theta} \gamma^{m} \hat{s}_{*}\right)\right]\left(\gamma_{m} \hat{\theta}\right)_{\hat{\alpha}}, \\
\delta_{\mathrm{BV}} \hat{w}_{i *}^{\hat{\alpha}}= & -\hat{\nabla}_{i} \hat{\lambda}^{\hat{\alpha}}+\hat{\Omega} \hat{w}_{i *}^{\hat{\alpha}}, \\
\delta_{\mathrm{BV}} \hat{A}_{*}^{i}= & -\hat{\lambda}^{\hat{\alpha}} \hat{w}_{\hat{\alpha}}^{i}-\epsilon^{i j} \partial_{j} \hat{B}-\hat{\Sigma} \epsilon^{i j} \hat{\chi}_{j}-\epsilon^{i j} \hat{s}_{\hat{\alpha}} \hat{w}_{j *}^{\hat{\alpha}}+\delta_{\tau}^{i} \hat{\theta}^{\hat{\alpha}} \hat{L}_{\hat{\alpha}}^{*}+\hat{\gamma} \hat{\chi}_{*}^{i}, \\
\delta_{\mathrm{BV}} \hat{B}^{*}= & -\epsilon^{i j} \partial_{i} \hat{A}_{j}, \\
\delta_{\mathrm{BV}} \hat{L}_{\hat{\alpha}}^{*}= & \frac{1}{4 \mathcal{T}}\left[P^{m}-\mathcal{T} \partial_{\sigma} X^{m}+\frac{1}{2}\left(\hat{\theta} \gamma^{m} \hat{w}_{\sigma *}\right)\right]\left(\gamma_{m} \hat{\lambda}\right)_{\hat{\alpha}}+\hat{\Omega} \hat{L}_{\hat{\alpha}}^{*}, \\
\delta_{\mathrm{BV}} \hat{\xi}_{\hat{\alpha}}^{*}= & \partial_{i} \hat{p}_{\hat{\alpha}}^{i}, \\
\delta_{\mathrm{BV}} \hat{p}_{i *}^{\hat{\alpha}}= & \partial_{i} \hat{\xi}^{\hat{\alpha}}-\hat{\lambda}^{\hat{\alpha}} \hat{\chi}_{i}+\hat{\gamma} \hat{w}_{i *}^{\hat{\alpha}}, \\
\delta_{\mathrm{BV}} \hat{\chi}_{*}^{i}= & \hat{\lambda}^{\hat{\alpha}} \hat{p}_{\hat{\alpha}}^{i}+\epsilon^{i j} \hat{\nabla}_{j} \hat{\Sigma}+\hat{\Omega} \hat{\chi}_{*}^{i}-\epsilon^{i j} \hat{\epsilon}_{\hat{\alpha}} \hat{w}_{j *}^{\hat{\alpha}}, \\
\delta_{\mathrm{BV}} \hat{\Sigma}^{*}= & -\epsilon^{i j} \hat{\nabla}_{i} \hat{\chi}_{j}-\hat{\Omega} \hat{\Sigma}^{*}+\hat{\gamma} \hat{B}^{*},
\end{aligned}
$$

and for the ghost antifields,

$$
\begin{aligned}
\delta_{\mathrm{BV}} \theta_{\alpha}^{*}= & \frac{1}{4 \mathcal{T}}\left[\eta^{m n} X_{n}^{*}-\mathcal{T} \partial_{\sigma} P_{*}^{m}-\frac{1}{4}\left(\theta \gamma^{m} s_{*}\right)\right]\left(\gamma_{m} \lambda\right)_{\alpha}+\Omega \theta_{\alpha}^{*}-\nabla_{\tau} L_{\alpha}^{*} \\
& +\frac{1}{8 \mathcal{T}}\left[\left(L \gamma_{m} \lambda\right)-\frac{1}{2}\left(w_{\tau *} \gamma_{m} \theta\right)\right]\left(\gamma^{m} w_{\sigma *}\right)_{\alpha}+\frac{1}{16 \mathcal{T}}\left(\lambda \gamma_{m} \theta\right)\left(\gamma^{m} s_{*}\right)_{\alpha} \\
& -\frac{1}{4 \mathcal{T}}\left[P_{m}+\mathcal{T} \partial_{\sigma} X_{m}+\frac{1}{4}\left(w_{\sigma *} \gamma_{m} \theta\right)\right]\left(\gamma^{m} w_{\tau *}\right)_{\alpha}, \\
\delta_{\mathrm{BV}} s_{*}^{\alpha}= & \epsilon^{i j} \nabla_{i} w_{j *}^{\alpha}-\lambda^{\alpha} B^{*}+\Omega s_{*}^{\alpha}, \\
\delta_{\mathrm{BV}} \Omega^{*}= & \lambda^{\alpha} \lambda_{\alpha}^{*}-w_{\alpha}^{i} w_{i *}^{\alpha}+\partial_{i} A_{*}^{i}-L^{\alpha} L_{\alpha}^{*}-\chi_{i} \chi_{*}^{i}+\Sigma \Sigma^{*}-\theta^{\alpha} \theta_{\alpha}^{*}-s_{\alpha} s_{*}^{\alpha}-\gamma \gamma^{*}, \\
\delta_{\mathrm{BV}} \gamma^{*}= & -p_{\alpha}^{i} w_{i *}^{\alpha}-\Sigma B^{*}-\lambda^{\alpha} \xi_{\alpha}^{*}+\nabla_{i} \chi_{*}^{i}+\Omega \gamma^{*}+\epsilon_{\alpha} s_{*}^{\alpha}, \\
\delta_{\mathrm{BV}} \epsilon_{*}^{\alpha}= & \epsilon^{i j} \chi_{i} w_{j *}^{\alpha}-\epsilon^{i j} \partial_{i} p_{j *}^{\alpha}+\lambda^{\alpha} \Sigma^{*}+\gamma s_{*}^{\alpha}, \\
\delta_{\mathrm{BV}} \hat{\theta}_{\hat{\alpha}}^{*}= & \frac{1}{4 \mathcal{T}}\left[\eta^{m n} X_{n}^{*}+\mathcal{T} \partial_{\sigma} P_{*}^{m}+\frac{1}{4}\left(\hat{\theta} \gamma^{m} \hat{s}_{*}\right)\right]\left(\gamma_{m} \hat{\lambda}\right)_{\hat{\alpha}}+\hat{\Omega} \hat{\theta}_{\hat{\alpha}}^{*}-\hat{\nabla}_{\tau} \hat{L}_{\hat{\alpha}}^{*} \\
& -\frac{1}{8 \mathcal{T}}\left[\left(\hat{L} \gamma_{m} \hat{\lambda}\right)-\frac{1}{2}\left(\hat{w}_{\tau *} \gamma_{m} \hat{\theta}\right)\right]\left(\gamma^{m} \hat{w}_{\sigma *}\right)_{\hat{\alpha}}-\frac{1}{16 \mathcal{T}}\left(\hat{\lambda} \gamma_{m} \hat{\theta}\right)\left(\gamma^{m} \hat{s}_{*}\right)_{\hat{\alpha}} \\
& -\frac{1}{4 \mathcal{T}}\left[P_{m}-\mathcal{T} \partial_{\sigma} X_{m}-\frac{1}{4}\left(\hat{w}_{\sigma *} \gamma_{m} \hat{\theta}\right)\right]\left(\gamma^{m} \hat{w}_{\tau *}\right)_{\hat{\alpha}}, \\
\delta_{\mathrm{BV}} \hat{s}_{*}^{\hat{\alpha}}= & \epsilon^{i j} \hat{\nabla}_{i} \hat{w}_{j *}^{\hat{\alpha}}-\hat{\lambda}^{\hat{\alpha}} \hat{B}^{*}+\hat{\Omega} \hat{s}_{*}^{\hat{\alpha}}, \\
\delta_{\mathrm{BV}} \hat{\Omega}^{*}= & \hat{\lambda}^{\hat{\alpha}} \hat{\lambda}_{\hat{\alpha}}^{*}-\hat{w}_{\hat{\alpha}}^{i} \hat{w}_{i *}^{\hat{\alpha}}+\partial_{i} \hat{A}_{*}^{i}-\hat{L}^{\hat{\alpha}} \hat{L}_{\hat{\alpha}}^{*}-\hat{\chi}_{i} \hat{\chi}_{*}^{i}+\hat{\Sigma} \hat{\Sigma}^{*}-\hat{\theta}^{\hat{\alpha}} \hat{\theta}_{\hat{\alpha}}^{*}-\hat{s}_{\hat{\alpha}} \hat{s}_{*}^{\hat{\alpha}}-\hat{\gamma} \hat{\gamma}^{*}, \\
\delta_{\mathrm{BV}} \hat{\gamma}^{*}= & -\hat{p}_{\hat{\alpha}}^{i} \hat{w}_{i *}^{\hat{\alpha}}-\hat{\Sigma} \hat{B}^{*}-\hat{\lambda}^{\hat{\alpha}} \hat{\xi}_{\hat{\alpha}}^{*}+\hat{\nabla}_{i} \hat{\chi}_{*}^{i}+\hat{\Omega} \hat{\gamma}^{*}+\hat{\epsilon}_{\hat{\alpha}} \hat{s}_{*}^{\hat{\alpha}}, \\
\delta_{\mathrm{BV}} \hat{\epsilon}_{*}^{\hat{\alpha}}= & \epsilon^{i j} \hat{\chi}_{i} \hat{w}_{j *}^{\hat{\alpha}}-\epsilon^{i j} \partial_{i} \hat{p}_{j *}^{\hat{\alpha}}+\hat{\lambda}^{\hat{\alpha}} \hat{\Sigma}^{*}+\hat{\gamma} \hat{s}_{*}^{\hat{\alpha}} .
\end{aligned}
$$




\subsection{Gauge fixing}

The procedure for gauge fixing in the Batalin-Vilkovisky formalism is very straightforward. But before moving on, it is useful to discuss the particular gauge to be chosen here.

Using the scaling transformations (2.24), it is possible to choose $A_{\tau}=\hat{A}_{\tau}=0$. Similarly, the fermionic gauge transformations (2.25) can be used to partially fix their gauge fields to $\chi_{\tau}=\hat{\chi}_{\tau}=0$.

For the curl symmetry $(2.26)$, the choice will be $w_{\alpha}^{\sigma}=p_{\alpha}^{\sigma}=0$ and $\hat{w}_{\hat{\alpha}}^{\sigma}=\hat{p}_{\hat{\alpha}}^{\sigma}=0$. Observe here that there is no residual gauge transformation, since both fields $\left(w_{\alpha}^{\sigma}, p_{\alpha}^{\sigma}, \hat{w}_{\hat{\alpha}}^{\sigma}\right.$ and $\left.\hat{p}_{\hat{\alpha}}^{\sigma}\right)$ and ghosts $\left(s_{\alpha}, \epsilon_{\alpha}, \hat{s}_{\hat{\alpha}}\right.$ and $\left.\hat{\epsilon}_{\hat{\alpha}}\right)$ have the same number of independent components according to the pure spinor constraints (2.34) and gauge transformations (2.36).

Finally, the twistor-like symmetry $(2.27)$ can be used to fix the Lagrange multipliers $L^{\alpha}$ and $\hat{L}^{\hat{\alpha}}$. Following the discussion after equation (2.17), the gauge $L^{\alpha}=\hat{L}^{\hat{\alpha}}=0$ would imply a degenerate worldsheet metric. Although worldsheet reparametrization is hidden in the twistor-like symmetry, the conformal gauge would still be the natural choice. In the Polyakov action (2.7), this gauge would be $e_{ \pm}=1$. For the action $S_{0}$ in (2.18), the conformal gauge is equivalent to the choice

$$
\begin{aligned}
L^{\alpha} & =\frac{\left(\gamma^{m} \Lambda\right)^{\alpha}}{(\Lambda \lambda)}\left(P_{m}+\mathcal{T} \partial_{\sigma} X_{m}\right), \\
\hat{L}^{\hat{\alpha}} & =\frac{\left(\gamma^{m} \hat{\Lambda}\right)^{\hat{\alpha}}}{(\hat{\Lambda} \hat{\lambda})}\left(P_{m}-\mathcal{T} \partial_{\sigma} X_{m}\right) .
\end{aligned}
$$

The proposed gauge can be implemented through the gauge fixing fermion $\Xi$, given by

$$
\begin{aligned}
\Xi= & \int d \tau d \sigma\left\{\bar{\Omega} A_{\tau}+\beta \chi_{\tau}-r^{\alpha} w_{\alpha}^{\sigma}+\eta^{\alpha} p_{\alpha}^{\sigma}+\hat{\bar{\Omega}}_{\tau}-\hat{r}^{\hat{\alpha}} \hat{w}_{\hat{\alpha}}^{\sigma}+\hat{\beta} \hat{\chi}_{\tau}+\hat{\eta}^{\hat{\alpha}} \hat{p}_{\hat{\alpha}}^{\sigma}\right\} \\
& -\int d \tau d \sigma\left\{\pi_{\alpha}\left[L^{\alpha}-\frac{\left(\gamma^{m} \Lambda\right)^{\alpha}}{(\Lambda \lambda)}\left(P_{m}+\mathcal{T} \partial_{\sigma} X_{m}\right)\right]+\hat{\pi}_{\hat{\alpha}}\left[\hat{L}^{\hat{\alpha}}-\frac{\left(\gamma^{m} \hat{\Lambda}\right)^{\hat{\alpha}}}{(\hat{\Lambda} \hat{\lambda})}\left(P_{m}-\mathcal{T} \partial_{\sigma} X_{m}\right)\right]\right\} .
\end{aligned}
$$

Here, $\bar{\Omega}, \beta, r^{\alpha}, \eta^{\alpha}$ and $\pi_{\alpha}$ are the antighosts of $\Omega, \gamma, s_{\alpha}, \epsilon_{\alpha}$ and $\theta^{\alpha}$, respectively (hatted and unhatted). The antighosts will be generically represented by $\bar{\Phi}_{a}$ (with antifields $\bar{\Phi}_{*}^{a}$ ), where the index $a$ denotes the different components of the gauge parameters. In addition, the extended phase space of the model will include the Nakanishi-Lautrup fields, $\Lambda_{a}$, and antifields, $\Lambda_{*}^{a}$.

The gauge fixed action can be determined by evaluating the non-minimal master action, defined as

$$
S_{n m}=S+\int d \tau d \sigma\left(\bar{\Phi}_{*}^{a} \Lambda_{a}\right)
$$

at

$$
\Phi_{I}^{*}=\frac{\delta \Xi}{\delta \Phi^{I}}, \quad \bar{\Phi}_{*}^{a}=\frac{\delta \Xi}{\delta \bar{\Phi}_{a}}, \quad \Lambda_{*}^{a}=\frac{\delta \Xi}{\delta \Lambda_{a}} .
$$


For the particular choice of gauge fixing fermion (2.47), the non-vanishing antifields are given by

$$
\begin{aligned}
X_{m}^{*} & =-\mathcal{T} \partial_{\sigma}\left[\frac{\left(\pi \gamma^{m} \Lambda\right)}{(\Lambda \lambda)}\right]+\mathcal{T} \partial_{\sigma}\left[\frac{\left(\hat{\pi} \gamma^{m} \hat{\Lambda}\right)}{(\hat{\Lambda} \hat{\lambda})}\right], & & A_{*}^{\tau}=\bar{\Omega}, \\
P_{*}^{m} & =\mathcal{T} \frac{\left(\pi \gamma^{m} \Lambda\right)}{(\Lambda \lambda)}+\mathcal{T} \frac{\left(\hat{\pi} \gamma^{m} \hat{\Lambda}\right)}{(\hat{\Lambda} \hat{\lambda})}, & \hat{A}_{*}^{\tau} & =\hat{\bar{\Omega}}, \\
\lambda_{\alpha}^{*} & =-\frac{\left(\pi \gamma^{m} \Lambda\right)}{(\Lambda \lambda)^{2}}\left(P_{m}+\mathcal{T} \partial_{\sigma} X_{m}\right) \Lambda_{\alpha}, & \chi_{*}^{\tau} & =\beta, \\
\hat{\lambda}_{\hat{\alpha}}^{*} & =-\frac{\left(\hat{\pi} \gamma^{m}\right)}{(\hat{\Lambda} \hat{\lambda})^{2}}\left(P_{m}-\mathcal{T} \partial_{\sigma} X_{m}\right) \hat{\Lambda}_{\hat{\alpha}}, & \hat{\chi}_{*}^{\tau} & =\hat{\beta}, \\
L_{\alpha}^{*} & =-\pi_{\alpha}, & \hat{L}_{\hat{\alpha}}^{*} & =-\hat{\pi}_{\hat{\alpha}}, \\
w_{\sigma *}^{\alpha} & =-r^{\alpha}, & \hat{w}_{\sigma *}^{\alpha} & =-\hat{r}^{\alpha}, \\
p_{\sigma *}^{\alpha} & =\eta^{\alpha}, & \hat{p}_{\sigma *}^{\alpha} & =\hat{\eta}^{\hat{\alpha}} .
\end{aligned}
$$

Since the master action $S$ was consistently built with the pure spinor constraints (2.34), the antighosts are constrained accordingly:

$$
\begin{aligned}
\xi^{\alpha} \pi_{\alpha} & =0, & \hat{\xi}^{\hat{\alpha}} \hat{\pi}_{\hat{\alpha}} & =0, \\
\lambda^{\alpha} \pi_{\alpha} & =0, & \hat{\lambda}^{\hat{\alpha}} \hat{\pi}_{\hat{\alpha}} & =0, \\
\left(\lambda \gamma^{m} r\right) & =0, & \left(\hat{\lambda} \gamma^{m} \hat{r}\right) & =0, \\
\left(\lambda \gamma^{m n} \pi\right) & =0, & \left(\hat{\lambda} \gamma^{m n} \hat{\pi}\right) & =0, \\
\left(\lambda \gamma^{m} \eta\right)+\left(r \gamma^{m} \xi\right) & =0, & \left(\hat{\lambda} \gamma^{m} \hat{\eta}\right)+\left(\hat{r} \gamma^{m} \hat{\xi}\right) & =0 .
\end{aligned}
$$

After solving for the equations of motion of the Nakanishi-Lautrup fields, the gauge fixed action can be written as

$$
\begin{aligned}
S_{\text {fixed }}= & \int d \tau d \sigma\left\{P_{m} \partial_{\tau} X^{m}-\frac{1}{2 \mathcal{T}}\left(P_{m} P^{m}+\mathcal{T}^{2} \partial_{\sigma} X_{m} \partial_{\sigma} X^{m}\right)\right\} \\
& +\int d \tau d \sigma\left\{w_{\alpha} \partial_{\tau} \lambda^{\alpha}+p_{\alpha} \partial_{\tau} \xi^{\alpha}+\pi_{\alpha} \partial_{\tau} \theta^{\alpha}+r^{\alpha} \partial_{\tau} s_{\alpha}+\eta^{\alpha} \partial_{\tau} \epsilon_{\alpha}\right\} \\
& +\int d \tau d \sigma\left\{A \partial_{\tau} B+\Sigma \partial_{\tau} \chi+\bar{\Omega} \partial_{\tau} \Omega+\beta \partial_{\tau} \gamma\right\} \\
& +\int d \tau d \sigma\left\{\frac{1}{2} \partial_{\sigma}\left(\lambda \gamma^{m} \theta\right) \frac{\left(\pi \gamma^{m} \Lambda\right)}{(\Lambda \lambda)}-\frac{1}{8 T} \frac{\left(\lambda \gamma^{n} \gamma^{m} \Lambda\right)}{(\Lambda \lambda)}\left(P_{m}+\mathcal{T} \partial_{\sigma} X_{m}\right)\left(\theta \gamma_{n} r\right)\right\} \\
& +\int d \tau d \sigma\left\{\hat{w}_{\hat{\alpha}} \partial_{\tau} \hat{\lambda}^{\hat{\alpha}}+\hat{p}_{\hat{\alpha}} \partial_{\tau} \hat{\xi}^{\hat{\alpha}}+\hat{\pi}_{\hat{\alpha}} \partial_{\tau} \hat{\theta}^{\hat{\alpha}}+\hat{r}^{\hat{\alpha}} \partial_{\tau} \hat{s}_{\hat{\alpha}}+\hat{\eta}^{\hat{\alpha}} \partial_{\tau} \hat{\epsilon}_{\hat{\alpha}}\right\} \\
& +\int d \tau d \sigma\left\{\hat{A} \partial_{\tau} \hat{B}+\hat{\Sigma} \partial_{\tau} \hat{\chi}+\hat{\bar{\Omega}} \partial_{\tau} \hat{\Omega}+\hat{\beta} \partial_{\tau} \hat{\gamma}\right\} \\
& +\int d \tau d \sigma\left\{-\frac{1}{2} \partial_{\sigma}\left(\hat{\lambda} \gamma^{m} \hat{\theta}\right) \frac{\left(\hat{\pi} \gamma^{m} \hat{\Lambda}\right)}{(\hat{\Lambda} \hat{\lambda})}+\frac{1}{8 T} \frac{\left(\hat{\lambda} \gamma^{n} \gamma^{m} \hat{\Lambda}\right)}{(\hat{\Lambda} \hat{\lambda})}\left(P_{m}-\mathcal{T} \partial_{\sigma} X_{m}\right)\left(\hat{\theta} \gamma_{n} \hat{r}\right)\right\}
\end{aligned}
$$

where some of the fields were renamed in order to simplify the notation,

$$
\begin{aligned}
& w_{\alpha} \equiv w_{\alpha}^{\tau}, A \equiv A_{\sigma}, \quad \hat{w}_{\hat{\alpha}} \equiv \hat{w}_{\hat{\alpha}}^{\tau}, \quad \hat{A} \equiv \hat{A}_{\sigma}, \\
& p_{\alpha} \equiv p_{\alpha}^{\tau}, \quad \chi \equiv \chi_{\sigma}, \quad \hat{p}_{\hat{\alpha}} \equiv \hat{p}_{\hat{\alpha}}^{\tau}, \quad \hat{\chi} \equiv \hat{\chi}_{\sigma} .
\end{aligned}
$$

The BV-BRST transformations can be readily determined next. From equation (2.41), the transformations of the fields can be written as

$$
\begin{aligned}
\delta X^{m} & =\frac{1}{4 \mathcal{T}}\left[\left(\lambda \gamma^{m} \theta\right)+\left(\hat{\lambda} \gamma^{m} \hat{\theta}\right)\right], \\
\delta P_{m} & =\frac{1}{4} \partial_{\sigma}\left[\left(\lambda \gamma_{m} \theta\right)-\left(\hat{\lambda} \gamma_{m} \hat{\theta}\right)\right],
\end{aligned}
$$




$$
\begin{aligned}
\delta \lambda^{\alpha} & =\Omega \lambda^{\alpha} \\
\delta w_{\alpha} & =\gamma p_{\alpha}-\epsilon_{\alpha} \chi-\Omega w_{\alpha}-\nabla_{\sigma} s_{\alpha}-\frac{1}{4 \mathcal{T}}\left[P_{m}+\mathcal{T} \partial_{\sigma} X_{m}-\frac{1}{4}\left(r \gamma_{m} \theta\right)\right]\left(\theta \gamma^{m}\right)_{\alpha} \\
\delta A & =-\partial_{\sigma} \Omega \\
\delta B & =\gamma \Sigma-\lambda^{\alpha} s_{\alpha} \\
\delta \xi^{\alpha} & =\gamma \lambda^{\alpha} \\
\delta p_{\alpha} & =-\partial_{\sigma} \epsilon_{\alpha} \\
\delta \chi & =\partial_{\sigma} \gamma-A \gamma-\Omega \chi \\
\delta \Sigma & =\Omega \Sigma-\lambda^{\alpha} \epsilon_{\alpha} \\
\delta \hat{\lambda}^{\hat{\alpha}} & =\hat{\Omega} \hat{\lambda}^{\hat{\alpha}} \\
\delta \hat{w}_{\hat{\alpha}} & =\hat{\gamma} \hat{p}_{\hat{\alpha}}-\hat{\epsilon}_{\hat{\alpha}} \hat{\chi}-\Omega \hat{w}_{\hat{\alpha}}-\hat{\nabla}_{\sigma} \hat{s}_{\hat{\alpha}}-\frac{1}{4 \mathcal{T}}\left[P_{m}-\mathcal{T} \partial_{\sigma} X_{m}+\frac{1}{4}\left(\hat{r} \gamma_{m} \hat{\theta}\right)\right]\left(\gamma^{m} \hat{\theta}\right)_{\hat{\alpha}} \\
\delta \hat{A} & =-\partial_{\sigma} \hat{\Omega} \\
\delta \hat{B} & =\hat{\gamma} \hat{\Sigma}-\hat{\lambda}^{\hat{\alpha}} \hat{s}_{\hat{\alpha}} \\
\delta \hat{\xi}^{\hat{\alpha}} & =\hat{\gamma} \hat{\lambda}^{\hat{\alpha}} \\
\delta \hat{p}_{\hat{\alpha}} & =-\partial_{\sigma} \hat{\epsilon}_{\hat{\alpha}} \\
\delta \hat{\chi} & =\partial_{\sigma} \hat{\gamma}-\hat{A} \hat{\gamma}-\hat{\Omega} \hat{\chi} \\
\delta \hat{\Sigma} & =\hat{\Omega} \hat{\Sigma}-\hat{\lambda}^{\hat{\alpha}} \hat{\epsilon}_{\hat{\alpha}}
\end{aligned}
$$

and, using equations (2.43) and (2.44), the transformations of the ghosts are given by

$$
\begin{aligned}
\delta \pi_{\alpha} & =\Omega \pi_{\alpha}-\frac{1}{4 \mathcal{T}}\left(\gamma_{m} \lambda\right)_{\alpha}\left(P^{m}+\mathcal{T} \partial_{\sigma} X^{m}\right)-\frac{1}{8 \mathcal{T}}\left(\gamma_{m} \lambda\right)_{\alpha}\left(\theta \gamma^{m} r\right), \\
\delta \theta^{\alpha} & =-\Omega \theta^{\alpha} \\
\delta r^{\alpha} & =\partial_{\sigma} \lambda^{\alpha}+A \lambda^{\alpha}+\Omega r^{\alpha} \\
\delta s_{\alpha} & =-\Omega s_{\alpha}-\gamma \epsilon_{\alpha}-\frac{1}{16 \mathcal{T}}\left(\lambda \gamma_{m} \theta\right)\left(\gamma^{m} \theta\right)_{\alpha}, \\
\delta \bar{\Omega} & =-\lambda^{\alpha} w_{\alpha}+\partial_{\sigma} B+r^{\alpha} s_{\alpha}+\pi_{\alpha} \theta^{\alpha}+\Sigma \chi+\beta \gamma, \\
\delta \Omega & =0 \\
\delta \beta & =\lambda^{\alpha} p_{\alpha}-\partial_{\sigma} \Sigma-A \Sigma+\Omega \beta-r^{\alpha} \epsilon_{\alpha}, \\
\delta \gamma & =-\Omega \gamma, \\
\delta \eta^{\alpha} & =\partial_{\sigma} \xi^{\alpha}-\lambda^{\alpha} \chi-\gamma r^{\alpha} \\
\delta \epsilon_{\alpha} & =0 \\
\delta \hat{\pi}_{\hat{\alpha}} & =\hat{\Omega} \hat{\pi}_{\hat{\alpha}}-\frac{1}{4 \mathcal{T}}\left(\gamma_{m} \hat{\lambda}\right)_{\hat{\alpha}}\left(P^{m}-\mathcal{T} \partial_{\sigma} X^{m}\right)+\frac{1}{8 \mathcal{T}}\left(\gamma_{m} \hat{\lambda}\right)_{\hat{\alpha}}\left(\hat{\theta} \gamma^{m} \hat{r}\right), \\
\delta \hat{\theta}^{\hat{\alpha}} & =-\hat{\Omega} \hat{\theta}^{\hat{\alpha}}, \\
\delta \hat{r}^{\hat{\alpha}} & =\partial_{\sigma} \hat{\lambda}^{\hat{\alpha}}+\hat{A} \hat{\lambda}^{\hat{\alpha}}+\hat{\Omega} r^{\hat{\alpha}}, \\
\delta \hat{s}_{\hat{\alpha}} & =-\hat{\Omega} \hat{s}_{\hat{\alpha}}-\hat{\gamma} \hat{\epsilon}_{\hat{\alpha}}+\frac{1}{16 \mathcal{T}}\left(\hat{\lambda} \gamma_{m} \hat{\theta}\right)\left(\gamma^{m} \hat{\theta}\right)_{\hat{\alpha}}, \\
\delta \hat{\bar{\Omega}} & =-\hat{\lambda}^{\hat{\alpha}} \hat{w}_{\hat{\alpha}}+\partial_{\sigma} \hat{B}+\hat{r}^{\hat{\alpha}} \hat{s}_{\hat{\alpha}}+\hat{\pi}_{\hat{\alpha}} \hat{\theta}^{\hat{\alpha}}+\hat{\Sigma} \hat{\chi}+\hat{\beta} \hat{\gamma}, \\
\delta \hat{\Omega} & =0
\end{aligned}
$$




$$
\begin{aligned}
\delta \hat{\beta} & =\hat{\lambda}^{\hat{\alpha}} \hat{p}_{\hat{\alpha}}-\partial_{\sigma} \hat{\Sigma}-\hat{A} \hat{\Sigma}+\hat{\Omega} \hat{\beta}-\hat{r}^{\hat{\alpha}} \hat{\epsilon}_{\hat{\alpha}}, \\
\delta \hat{\gamma} & =-\hat{\Omega} \hat{\gamma}, \\
\delta \hat{\eta}^{\hat{\alpha}} & =\partial_{\sigma} \hat{\xi}^{\hat{\alpha}}-\hat{\lambda}^{\hat{\alpha}} \hat{\chi}-\hat{\gamma} \hat{r}^{\hat{\alpha}}, \\
\delta \hat{\epsilon}_{\hat{\alpha}} & =0 .
\end{aligned}
$$

The action (2.52) does not look like an ordinary action in the conformal gauge. This can be fixed with the addition of a BRST trivial expression. Consider the operators

$$
\begin{gathered}
I \equiv-\frac{1}{2}\left(r \gamma^{m} \theta\right) \frac{\left(\pi \gamma^{m} \Lambda\right)}{(\Lambda \lambda)}+w_{\alpha} r^{\alpha}-p_{\alpha} \eta^{\alpha}+A \bar{\Omega}+\chi \beta, \\
\hat{I} \equiv \frac{1}{2}\left(\hat{r} \gamma^{m} \hat{\theta}\right) \frac{\left(\hat{\pi} \gamma^{m} \hat{\Lambda}\right)}{(\hat{\Lambda} \hat{\lambda})}+\hat{w}_{\hat{\alpha}} \hat{r}^{\hat{\alpha}}-\hat{p}_{\hat{\alpha}} \hat{\eta}^{\hat{\alpha}}+\hat{A} \hat{\bar{\Omega}}+\hat{\chi} \hat{\beta},
\end{gathered}
$$

and their BRST variation. It is then straightforward to demonstrate that

$$
\begin{aligned}
\tilde{S} \equiv & S_{\text {fixed }}+\left\{Q, \int d \tau d \sigma(I+\hat{I})\right\} \\
= & \int d \tau d \sigma\left\{P_{m} \partial_{\tau} X^{m}-\frac{1}{2 \mathcal{T}}\left(P_{m} P^{m}+\mathcal{T}^{2} \partial_{\sigma} X_{m} \partial_{\sigma} X^{m}\right)\right\} \\
& +\int d \tau d \sigma\left\{w_{\alpha} \partial_{-} \lambda^{\alpha}+p_{\alpha} \partial_{-} \xi^{\alpha}+\pi_{\alpha} \partial_{\tau} \theta^{\alpha}+r^{\alpha} \partial_{-} s_{\alpha}+\eta^{\alpha} \partial_{-} \epsilon_{\alpha}\right\} \\
& +\int d \tau d \sigma\left\{A \partial_{-} B+\Sigma \partial_{-} \chi+\bar{\Omega} \partial_{-} \Omega+\beta \partial_{-} \gamma+\frac{1}{2}\left(\lambda \gamma^{m} \partial_{\sigma} \theta\right) \frac{\left(\pi \gamma^{m} \Lambda\right)}{(\Lambda \lambda)}\right\} \\
& +\int d \tau d \sigma\left\{\hat{w}_{\hat{\alpha}} \partial_{+} \hat{\lambda}^{\hat{\alpha}}+\hat{p}_{\hat{\alpha}} \partial_{+} \hat{\xi}^{\hat{\alpha}}+\hat{\pi}_{\hat{\alpha}} \partial_{\tau} \hat{\theta}^{\hat{\alpha}}+\hat{r}^{\hat{\alpha}} \partial_{+} \hat{s}_{\hat{\alpha}}+\hat{\eta}^{\hat{\alpha}} \partial_{+} \hat{\epsilon}_{\hat{\alpha}}\right\} \\
& +\int d \tau d \sigma\left\{\hat{A} \partial_{+} \hat{B}+\hat{\Sigma} \partial_{+} \hat{\chi}+\hat{\bar{\Omega}} \partial_{+} \hat{\Omega}+\hat{\beta} \partial_{+} \hat{\gamma}-\frac{1}{2}\left(\hat{\lambda} \gamma^{m} \partial_{\sigma} \hat{\theta}\right) \frac{\left(\hat{\pi} \gamma^{m} \hat{\Lambda}\right)}{(\hat{\Lambda} \hat{\lambda})}\right\},
\end{aligned}
$$

where $\partial_{ \pm}=\partial_{\tau} \pm \partial_{\sigma}$. Now, using the gamma matrix identity

$$
\left(\gamma^{m n}\right)_{\alpha}^{\beta}\left(\gamma_{m n}\right)_{\lambda}^{\gamma}=4 \gamma_{\alpha \lambda}^{m} \gamma_{m}^{\beta \gamma}-2 \delta_{\alpha}^{\beta} \delta_{\lambda}^{\gamma}-8 \delta_{\lambda}^{\beta} \delta_{\alpha}^{\gamma},
$$

it follows that

$$
\begin{aligned}
-\frac{1}{2}\left(\lambda \gamma_{m} \partial_{\sigma} \theta\right)\left(\pi \gamma^{m} \Lambda\right) & =\frac{1}{8}\left(\pi \gamma^{m n} \lambda\right)\left(\Lambda \gamma_{m n} \partial_{\sigma} \theta\right)+\frac{1}{4}(\pi \lambda)\left(\Lambda \partial_{\sigma} \theta\right)+(\Lambda \lambda)\left(\pi \partial_{\sigma} \theta\right), \\
-\frac{1}{2}\left(\hat{\lambda} \gamma_{m} \partial_{\sigma} \hat{\theta}\right)\left(\hat{\pi} \gamma^{m} \hat{\Lambda}\right) & =\frac{1}{8}\left(\hat{\pi} \gamma^{m n} \hat{\lambda}\right)\left(\hat{\Lambda} \gamma_{m n} \partial_{\sigma} \hat{\theta}\right)+\frac{1}{4}(\hat{\pi} \hat{\lambda})\left(\hat{\Lambda} \partial_{\sigma} \hat{\theta}\right)+(\hat{\Lambda} \hat{\lambda})\left(\hat{\pi} \partial_{\sigma} \hat{\theta}\right) .
\end{aligned}
$$

The first two terms on the right hand side of each equation vanish due to the con- 
straints (2.51). Therefore the gauge fixed action (2.57) can be finally rewritten as

$$
\begin{aligned}
\tilde{S}= & \int d \tau d \sigma\left\{P_{m} \partial_{\tau} X^{m}-\frac{1}{2 \mathcal{T}}\left(P_{m} P^{m}+\mathcal{T}^{2} \partial_{\sigma} X_{m} \partial_{\sigma} X^{m}\right)\right\} \\
& +\int d \tau d \sigma\left\{w_{\alpha} \partial_{-} \lambda^{\alpha}+p_{\alpha} \partial_{-} \xi^{\alpha}+\pi_{\alpha} \partial_{-} \theta^{\alpha}+r^{\alpha} \partial_{-} s_{\alpha}+\eta^{\alpha} \partial_{-} \epsilon_{\alpha}\right\} \\
& +\int d \tau d \sigma\left\{A \partial_{-} B+\Sigma \partial_{-} \chi+\bar{\Omega} \partial_{-} \Omega+\beta \partial_{-} \gamma\right\} \\
& +\int d \tau d \sigma\left\{\hat{w}_{\hat{\alpha}} \partial_{+} \hat{\lambda}^{\hat{\alpha}}+\hat{p}_{\hat{\alpha}} \partial_{+} \hat{\xi}^{\hat{\alpha}}+\hat{\pi}_{\hat{\alpha}} \partial_{+} \hat{\theta}^{\hat{\alpha}}+\hat{r}^{\hat{\alpha}} \partial_{+} \hat{s}_{\hat{\alpha}}+\hat{\eta}^{\hat{\alpha}} \partial_{+} \hat{\epsilon}_{\hat{\alpha}}\right\} \\
& +\int d \tau d \sigma\left\{\hat{A} \partial_{+} \hat{B}+\hat{\Sigma} \partial_{+} \hat{\chi}+\hat{\bar{\Omega}} \partial_{+} \hat{\Omega}+\hat{\beta} \partial_{+} \hat{\gamma}\right\},
\end{aligned}
$$

in which the separation between right and left-moving sectors is manifest.

The BRST current can be computed using the transformations (2.54) and (2.55). It has two components, one left and one right-moving, given by

$$
\begin{aligned}
J= & -\frac{1}{4 \mathcal{T}}\left(\lambda \gamma_{m} \theta\right)\left(P^{m}+\mathcal{T} \partial_{\sigma} X^{m}\right)+\frac{1}{16 \mathcal{T}}\left(r \gamma^{m} \theta\right)\left(\lambda \gamma_{m} \theta\right)-\lambda^{\alpha} \partial_{\sigma} s_{\alpha} \\
& +A \lambda^{\alpha} s_{\alpha}-\Omega\left(\lambda^{\alpha} w_{\alpha}-r^{\alpha} s_{\alpha}+\theta^{\alpha} \pi_{\alpha}-\partial_{\sigma} B-\Sigma \chi-\beta \gamma\right) \\
& +\gamma\left(\lambda^{\alpha} p_{\alpha}-r^{\alpha} \epsilon_{\alpha}-\partial_{\sigma} \Sigma-A \Sigma\right)+\epsilon_{\alpha} \partial_{\sigma} \xi^{\alpha}-\chi \lambda^{\alpha} \epsilon_{\alpha}, \\
\hat{J}= & -\frac{1}{4 \mathcal{T}}\left(\hat{\lambda} \gamma_{m} \hat{\theta}\right)\left(P^{m}-\mathcal{T} \partial_{\sigma} X^{m}\right)-\frac{1}{16 \mathcal{T}}\left(\hat{r} \gamma^{m} \hat{\theta}\right)\left(\hat{\lambda} \gamma_{m} \hat{\theta}\right)-\hat{\lambda}^{\hat{\alpha}} \partial_{\sigma} \hat{s}_{\alpha} \\
& +\hat{A} \hat{\lambda}^{\hat{\alpha}} \hat{s}_{\alpha}-\hat{\Omega}\left(\hat{\lambda}^{\hat{\alpha}} \hat{w}_{\hat{\alpha}}+\hat{\theta}^{\hat{\alpha}} \hat{\pi}_{\hat{\alpha}}-\hat{r}^{\hat{\alpha}} \hat{s}_{\hat{\alpha}}-\partial_{\sigma} \hat{B}-\hat{\Sigma} \hat{\chi}-\hat{\beta} \hat{\gamma}\right) \\
& +\hat{\gamma}\left(\hat{\lambda}^{\hat{\alpha}} \hat{p}_{\hat{\alpha}}-\hat{r}^{\hat{\alpha}} \hat{\epsilon}_{\hat{\alpha}}-\partial_{\sigma} \hat{\Sigma}-\hat{A} \hat{\Sigma}\right)+\hat{\epsilon}_{\hat{\alpha}} \partial_{\sigma} \hat{\xi}^{\hat{\alpha}}-\hat{\chi} \hat{\lambda}^{\hat{\alpha}} \hat{\epsilon}_{\hat{\alpha}},
\end{aligned}
$$

such that $\partial_{-} J=\partial_{+} \hat{J}=0$.

As one final consistency check, observe that the generators of reparametrization symmetry are exact. By defining,

$$
\begin{aligned}
& B_{+} \equiv \frac{\left(\Lambda \gamma^{m} \pi\right)}{(\Lambda \lambda)}\left[P_{m}+\mathcal{T} \partial_{\sigma} X_{m}-\frac{1}{2}\left(r \gamma_{m} \theta\right)\right]-r^{\alpha} w_{\alpha}+\eta^{\alpha} p_{\alpha}-\beta \chi-A \bar{\Omega} \\
& B_{-} \equiv-\frac{\left(\hat{\Lambda} \gamma^{m} \hat{\pi}\right)}{(\hat{\Lambda} \hat{\lambda})}\left[P_{m}-\mathcal{T} \partial_{\sigma} X_{m}+\frac{1}{2}\left(\hat{r} \gamma_{m} \hat{\theta}\right)\right]-\hat{r}^{\hat{\alpha}} \hat{w}_{\hat{\alpha}}+\hat{\eta}^{\hat{\alpha}} \hat{p}_{\hat{\alpha}}-\hat{\beta} \hat{\chi}-\hat{A} \hat{\bar{\Omega}}
\end{aligned}
$$

it is straightforward to compute their BV-BRST transformation, cf. eqs. (2.54) and (2.55),

$$
\begin{aligned}
\delta B_{+}= & -\frac{1}{4 \mathcal{T}}\left(P_{m}+\mathcal{T} \partial_{\sigma} X_{m}\right)\left(P^{m}+\mathcal{T} \partial_{\sigma} X^{m}\right)-w_{\alpha} \partial_{\sigma} \lambda^{\alpha}-p_{\alpha} \partial_{\sigma} \xi^{\alpha}-A \partial_{\sigma} B \\
& -\chi \partial_{\sigma} \Sigma-\pi_{\alpha} \partial_{\sigma} \theta^{\alpha}-r^{\alpha} \partial_{\sigma} s_{\alpha}-\eta^{\alpha} \partial_{\sigma} \epsilon_{\alpha}-\beta \partial_{\sigma} \gamma-\bar{\Omega} \partial_{\sigma} \Omega, \\
\delta B_{-}= & \frac{1}{4 \mathcal{T}}\left(P^{m}-\mathcal{T} \partial_{\sigma} X^{m}\right)\left(P_{m}-\mathcal{T} \partial_{\sigma} X_{m}\right)-\hat{w}_{\hat{\alpha}} \partial_{\sigma} \hat{\lambda}^{\hat{\alpha}}-\hat{p}_{\hat{\alpha}} \partial_{\sigma} \hat{\xi}^{\hat{\alpha}}-\hat{A} \partial_{\sigma} \hat{B} \\
& -\hat{\chi} \partial_{\sigma} \hat{\Sigma}-\hat{\pi}_{\hat{\alpha}} \partial_{\sigma} \hat{\theta}^{\hat{\alpha}}-\hat{r}^{\hat{\alpha}} \partial_{\sigma} \hat{s}_{\hat{\alpha}}-\hat{\eta}^{\hat{\alpha}} \partial_{\sigma} \hat{\epsilon}_{\hat{\alpha}}-\hat{\beta} \partial_{\sigma} \hat{\gamma}-\hat{\bar{\Omega}} \partial_{\sigma} \hat{\Omega},
\end{aligned}
$$

which constitute the generalization of $H^{ \pm}$in (2.12). 


\subsection{Field redefinition and emergent spacetime supersymmetry}

Although not obviously, the BRST structure of the action (2.60) can be greatly simplified. Consider the field redefinitions

$$
\begin{array}{rlrl}
\lambda^{\alpha} & \rightarrow \gamma^{-1} \lambda^{\alpha}, & p_{\alpha} & \rightarrow p_{\alpha}+\partial_{\sigma} s_{\alpha}, \\
w_{\alpha} \rightarrow \gamma w_{\alpha}, & \pi_{\alpha} \rightarrow \gamma^{-1} \pi_{\alpha}, \\
A \rightarrow A+\frac{\partial_{\sigma} \gamma}{\gamma}, & r^{\alpha} \rightarrow \gamma^{-1}\left(r^{\alpha}+\partial_{\sigma} \xi^{\alpha}\right), \\
\chi & \rightarrow \gamma \chi, & s_{\alpha} \rightarrow \gamma s_{\alpha}, \\
\Sigma \rightarrow \gamma^{-1} \Sigma, & \beta \rightarrow \beta+\gamma^{-1}\left(\lambda^{\alpha} w_{\alpha}+\theta^{\alpha} \pi_{\alpha}-\partial_{\sigma} B\right) \\
\theta^{\alpha} \rightarrow \gamma \theta^{\alpha}, & & & +\gamma^{-1}\left(s_{\alpha} r^{\alpha}+s_{\alpha} \partial_{\sigma} \xi^{\alpha}+\chi \Sigma\right),
\end{array}
$$

and analogous operations in the hatted sector, which leave the action (2.60) invariant. Note that the pure spinor constraints (2.51) have to be modified accordingly.

Since the ghosts fields $\gamma$ and $\hat{\gamma}$ transform under scaling, the field redefinitions above leave all the spacetime spinors scale invariant at the price of shifting their ghost number. In particular, the pure spinors $\lambda^{\alpha}$ and $\hat{\lambda}^{\hat{\alpha}}$ now have ghost number +1 while the $\theta^{\alpha}$ and $\hat{\theta}^{\hat{\alpha}}$ have ghost number zero. Also, due to the ordering of the operators, the scaling parts of the BRST current (with $\Omega$ and $\hat{\Omega}$ ) receive quantum corrections which can be cast as

$$
\begin{aligned}
& \Omega\left(\lambda^{\alpha} w_{\alpha}-r^{\alpha} s_{\alpha}+\theta^{\alpha} \pi_{\alpha}-\partial_{\sigma} B-\Sigma \chi-\beta \gamma\right) \rightarrow-\Omega\left(\beta \gamma+c_{\#} \partial_{\sigma} \ln \gamma\right), \\
& \hat{\Omega}\left(\hat{\lambda}^{\hat{\alpha}} \hat{w}_{\hat{\alpha}}+\hat{\theta}^{\hat{\alpha}} \hat{\pi}_{\hat{\alpha}}-\hat{r}^{\hat{\alpha}} \hat{s}_{\hat{\alpha}}-\partial_{\sigma} \hat{B}-\hat{\Sigma} \hat{\chi}-\hat{\beta} \hat{\gamma}\right) \rightarrow-\hat{\Omega}\left(\hat{\beta} \hat{\gamma}+\hat{c}_{\#} \partial_{\sigma} \ln \hat{\gamma}\right),
\end{aligned}
$$

where $c_{\#}$ and $\hat{c}_{\#}$ are numerical constants which will be fixed later in appendix B.

The BRST currents (2.61) are then rewritten as

$$
\begin{aligned}
J= & -\frac{1}{4 \mathcal{T}}\left(\lambda \gamma_{m} \theta\right)\left[P^{m}+\mathcal{T} \partial_{\sigma} X^{m}-\frac{1}{4}\left(\partial_{\sigma} \xi \gamma^{m} \theta\right)\right] \\
& +\lambda^{\alpha} p_{\alpha}-\Omega\left(\beta \gamma+c_{\#} \partial_{\sigma} \ln \gamma\right)-A \Sigma-r^{\alpha} \epsilon_{\alpha} \\
& +\frac{1}{16 \mathcal{T}}\left(r \gamma^{m} \theta\right)\left(\lambda \gamma_{m} \theta\right)+A \lambda^{\alpha} s_{\alpha}-\chi \lambda^{\alpha} \epsilon_{\alpha}-\partial_{\sigma}(\gamma \Sigma), \\
\hat{J}= & -\frac{1}{4 \mathcal{T}}\left(\hat{\lambda} \gamma_{m} \hat{\theta}\right)\left[P^{m}-\mathcal{T} \partial_{\sigma} X^{m}+\frac{1}{4}\left(\partial_{\sigma} \hat{\xi} \gamma^{m} \hat{\theta}\right)\right] \\
& +\hat{\lambda}^{\hat{\alpha}} \hat{p}_{\hat{\alpha}}-\hat{\Omega}\left(\hat{\beta} \hat{\gamma}+\hat{c}_{\#} \partial_{\sigma} \ln \hat{\gamma}\right)-\hat{A} \hat{\Sigma}-\hat{r}^{\hat{\alpha}} \hat{\epsilon}_{\hat{\alpha}} \\
& -\frac{1}{16 \mathcal{T}}\left(\hat{r} \gamma^{m} \hat{\theta}\right)\left(\hat{\lambda} \gamma_{m} \hat{\theta}\right)+\hat{A} \hat{\lambda}^{\hat{\alpha}} \hat{s}_{\alpha}-\hat{\chi} \hat{\lambda}^{\hat{\alpha}} \hat{\epsilon}_{\hat{\alpha}}-\partial_{\sigma}(\hat{\gamma} \hat{\Sigma}) .
\end{aligned}
$$

The last term in each current can be disregarded, since they correspond to total derivatives and do not contribute to the BRST charges. Furthermore, the remaining terms in the third lines of (2.66a) and (2.66b) can be removed by similarity transformations of the form $J^{\prime} \equiv e^{-U} J e^{U}$ and $\hat{J}^{\prime} \equiv e^{-\hat{U}} \hat{J} e^{\hat{U}}$, where $U$ and $\hat{U}$ are invariant under the pure spinor symmetries and given by

$$
\begin{aligned}
& U=\int d \sigma\left\{\chi \lambda^{\alpha} s_{\alpha}-\frac{1}{32 \mathcal{T}}\left(\lambda \gamma^{m} \theta\right)\left(\lambda \gamma^{n} \theta\right) \frac{\left(\eta \gamma_{m n} \Lambda\right)}{(\Lambda \lambda)}\right\}, \\
& \hat{U}=\int d \sigma\left\{\hat{\chi} \hat{\lambda}^{\hat{\alpha}} \hat{s}_{\hat{\alpha}}+\frac{1}{32 \mathcal{T}}\left(\hat{\lambda} \gamma^{m} \hat{\theta}\right)\left(\hat{\lambda} \gamma^{n} \hat{\theta}\right) \frac{\left(\hat{\eta} \gamma_{m n} \hat{\Lambda}\right)}{(\hat{\Lambda} \hat{\lambda})}\right\},
\end{aligned}
$$


It is then straightforward to show that

$$
\begin{aligned}
J^{\prime}= & \lambda^{\alpha} p_{\alpha}-\frac{1}{4 \mathcal{T}}\left(\lambda \gamma_{m} \theta\right)\left[P^{m}+\mathcal{T} \partial_{\sigma} X^{m}-\frac{1}{4}\left(\partial_{\sigma} \xi \gamma^{m} \theta\right)\right] \\
& -\Omega\left(\beta \gamma+c_{\#} \partial_{\sigma} \ln \gamma\right)-A \Sigma-r^{\alpha} \epsilon_{\alpha}, \\
\hat{J}^{\prime}= & \hat{\lambda}^{\hat{\alpha}} \hat{p}_{\hat{\alpha}}-\frac{1}{4 \mathcal{T}}\left(\hat{\lambda} \gamma_{m} \hat{\theta}\right)\left[P^{m}-\mathcal{T} \partial_{\sigma} X^{m}+\frac{1}{4}\left(\partial_{\sigma} \hat{\xi} \gamma^{m} \hat{\theta}\right)\right] \\
& -\hat{\Omega}\left(\hat{\beta} \hat{\gamma}+\hat{c}_{\#} \partial_{\sigma} \ln \hat{\gamma}\right)-\hat{A} \hat{\Sigma}-\hat{r}^{\hat{\alpha}} \hat{\epsilon}_{\hat{\alpha}} .
\end{aligned}
$$

It is important to emphasize that the field redefinitions in (2.64) are well defined only if $\gamma$ and $\hat{\gamma}$ are assumed to be non-vanishing in every point of the worldsheet. This is clear in the definition of the conformal field theory of the decoupled sector, which is singular for $\gamma=0$ and $\hat{\gamma}=0$. In a path integral formulation, this singularity can be avoided by choosing a convenient parametrization for the ghosts, e.g. $\gamma=e^{\sigma}$ and $\beta=\rho e^{-\sigma}$, therefore enforcing the non-vanishing condition. Here, $\sigma$ is a chiral worldsheet scalar with conjugate $\rho$. More details can be found in the appendix B.

Using the quartet argument, the BRST cohomology can be shown to be independent of $A, B, \chi, \sigma, \rho, \Sigma, r^{\alpha}, s_{\alpha}, \eta^{\alpha}$ and $\epsilon_{\alpha}$ (hatted and unhatted). Therefore, these fields can be eliminated from the theory and the gauge fixed action (2.60) is further simplified to

$$
\begin{aligned}
\tilde{S}= & \int d \tau d \sigma\left\{P_{m} \partial_{\tau} X^{m}-\frac{1}{2 \mathcal{T}}\left(P_{m} P^{m}+\mathcal{T}^{2} \partial_{\sigma} X_{m} \partial_{\sigma} X^{m}\right)\right\} \\
& +\int d \tau d \sigma\left\{w_{\alpha} \partial_{-} \lambda^{\alpha}+p_{\alpha} \partial_{-} \xi^{\alpha}+\pi_{\alpha} \partial_{-} \theta^{\alpha}+\hat{w}_{\hat{\alpha}} \partial_{+} \hat{\lambda}^{\hat{\alpha}}+\hat{p}_{\hat{\alpha}} \partial_{+} \hat{\xi}^{\hat{\alpha}}+\hat{\pi}_{\hat{\alpha}} \partial_{+} \hat{\theta}^{\hat{\alpha}}\right\} .
\end{aligned}
$$

The pure spinor constraints (2.34) are reduced to

$$
\begin{aligned}
\left(\lambda \gamma^{m} \lambda\right) & =0, & \left(\hat{\lambda} \gamma^{m} \hat{\lambda}\right) & =0, \\
\left(\lambda \gamma^{m} \xi\right) & =0, & \left(\hat{\lambda} \gamma^{m} \hat{\xi}\right) & =0, \\
\xi^{\alpha} \pi_{\alpha} & =0, & \hat{\xi}^{\hat{\alpha}} \hat{\pi}_{\hat{\alpha}} & =0, \\
\lambda^{\alpha} \pi_{\alpha} & =0, & \hat{\lambda}^{\hat{\alpha}} \hat{\pi}_{\hat{\alpha}} & =0, \\
\left(\lambda \gamma^{m n} \pi\right) & =0, & \left(\hat{\lambda} \gamma^{m n} \hat{\pi}\right) & =0,
\end{aligned}
$$

and the action (2.69) is invariant under the implied pure spinor gauge transformations

$$
\begin{aligned}
\delta w_{\alpha} & =d_{m}\left(\gamma^{m} \lambda\right)_{\alpha}+e_{m}\left(\gamma^{m} \xi\right)_{\alpha}-\bar{f} \pi_{\alpha}-\bar{f}_{m n}\left(\gamma^{m n} \pi\right)_{\alpha}, \\
\delta p_{\alpha} & =e_{m}\left(\gamma^{m} \lambda\right)_{\alpha}-\bar{g} \pi_{\alpha}, \\
\delta \theta^{\alpha} & =\bar{f} \lambda^{\alpha}+\bar{f}_{m n}\left(\gamma^{m n} \lambda\right)^{\alpha}+\bar{g} \xi^{\alpha} \\
\delta \hat{w}_{\hat{\alpha}} & =\hat{d}_{m}\left(\gamma^{m} \hat{\lambda}\right)_{\hat{\alpha}}+\hat{e}_{m}\left(\gamma^{m} \hat{\xi}\right)_{\hat{\alpha}}-\hat{\bar{f}}_{\hat{\alpha}}-\hat{\bar{f}}_{m n}\left(\gamma^{m n} \hat{\pi}\right)_{\hat{\alpha}}, \\
\delta \hat{p}_{\hat{\alpha}} & =\hat{e}_{m}\left(\gamma^{m} \hat{\lambda}\right)_{\hat{\alpha}}-\hat{\bar{g}} \hat{\pi}_{\hat{\alpha}}, \\
\delta \hat{\theta}^{\hat{\alpha}} & =\hat{\bar{f}} \hat{\lambda}^{\hat{\alpha}}+\hat{\bar{f}}_{m n}\left(\gamma^{m n} \hat{\lambda}\right)^{\hat{\alpha}}+\hat{\bar{g}} \hat{\xi}^{\hat{\alpha}} .
\end{aligned}
$$

These transformations are the key to spacetime supersymmetry. The parameter $e_{m}$ can be tuned in such a way that the gauge dependent components of $p_{\alpha}$ are identified with the independent components of the constrained spinor $\pi_{\alpha}$. Furthermore, the gauge 
parameters $\bar{f}$ and $\bar{f}_{m n}$ can be similarly chosen such that the gauge dependent components of $\theta^{\alpha}$ are identified with the independent components of the constrained spinor $\xi^{\alpha}$. This is demonstrated in appendix A. An analogous gauge fixing can be performed in the hatted sector. The outcome of the partial gauge fixing of the pure spinor symmetries is the action

$$
\begin{aligned}
S= & \int d \tau d \sigma\left\{P_{m} \partial_{\tau} X^{m}-\frac{1}{2 \mathcal{T}}\left(P_{m} P^{m}+\mathcal{T}^{2} \partial_{\sigma} X_{m} \partial_{\sigma} X^{m}\right)\right\} \\
& +\int d \tau d \sigma\left\{w_{\alpha} \partial_{-} \lambda^{\alpha}+p_{\alpha} \partial_{-} \theta^{\alpha}+\hat{w}_{\hat{\alpha}} \partial_{+} \hat{\lambda}^{\hat{\alpha}}+\hat{p}_{\hat{\alpha}} \partial_{+} \hat{\theta}^{\hat{\alpha}}\right\},
\end{aligned}
$$

but now with unconstrained $p_{\alpha}, \theta^{\alpha}, \hat{p}_{\hat{\alpha}}$ and $\hat{\theta}^{\hat{\alpha}}$. The action $S$ corresponds to the type II-B superstring in the pure spinor formalism. The type II-A is similarly obtained by reverting the spinor chirality of one of the sectors, either hatted or unhatted. The heterotic superstring is obtained when only one of the twistor-like constraints (2.13) is imposed, but then worldsheet reparametrization has be taken into account in the construction of the master action.

The non-vanishing BRST transformations can be cast as

$$
\begin{array}{rlrl}
\delta X^{m}= & \frac{1}{4 \mathcal{T}}\left(\lambda \gamma^{m} \theta\right)+\frac{1}{4 \mathcal{T}}\left(\hat{\lambda} \gamma^{m} \hat{\theta}\right), & \delta P_{m}= & \frac{1}{4} \partial_{\sigma}\left[\left(\lambda \gamma_{m} \theta\right)\right]-\frac{1}{4} \partial_{\sigma}\left[\left(\hat{\lambda} \gamma_{m} \hat{\theta}\right)\right], \\
\delta w_{\alpha}= & p_{\alpha}-\frac{1}{16 \mathcal{T}}\left(\theta \gamma_{m} \partial_{\sigma} \theta\right)\left(\gamma^{m} \theta\right)_{\alpha} & \delta \hat{w}_{\hat{\alpha}}=\hat{p}_{\hat{\alpha}}+\frac{1}{16 \mathcal{T}}\left(\hat{\theta} \gamma_{m} \partial_{\sigma} \hat{\theta}\right)\left(\gamma^{m} \hat{\theta}\right)_{\hat{\alpha}} \\
& -\frac{1}{4 \mathcal{T}}\left(P_{m}+\mathcal{T} \partial_{\sigma} X_{m}\right)\left(\gamma^{m} \theta\right)_{\alpha}, & & -\frac{1}{4 \mathcal{T}}\left(P_{m}-\mathcal{T} \partial_{\sigma} X_{m}\right)\left(\gamma^{m} \hat{\theta}\right)_{\hat{\alpha}}, \\
\delta \theta^{\alpha}= & \lambda^{\alpha}, & \delta \hat{\theta}^{\hat{\alpha}}=\hat{\lambda}^{\hat{\alpha}}, \\
\delta p_{\alpha}= & -\frac{1}{4 \mathcal{T}}\left(P_{m}+\mathcal{T} \partial_{\sigma} X_{m}\right)\left(\gamma^{m} \lambda\right)_{\alpha} & \delta \hat{p}_{\hat{\alpha}}= & -\frac{1}{4 \mathcal{T}}\left(P_{m}-\mathcal{T} \partial_{\sigma} X_{m}\right)\left(\gamma^{m} \hat{\lambda}\right)_{\hat{\alpha}} \\
& +\frac{1}{16 \mathcal{T}}\left(\partial_{\sigma} \lambda \gamma_{m} \theta\right)\left(\gamma^{m} \theta\right)_{\alpha} & & -\frac{1}{16 \mathcal{T}}\left(\partial_{\sigma} \hat{\lambda} \gamma_{m} \hat{\theta}\right)\left(\gamma^{m} \hat{\theta}\right)_{\hat{\alpha}} \\
& +\frac{3}{8 \mathcal{T}}\left(\lambda \gamma_{m} \theta\right)\left(\gamma^{m} \partial_{\sigma} \theta\right)_{\alpha} & & -\frac{3}{8 \mathcal{T}}\left(\hat{\lambda} \gamma_{m} \hat{\theta}\right)\left(\gamma^{m} \partial_{\sigma} \hat{\theta}\right)_{\hat{\alpha}}
\end{array}
$$

generated by the BRST charges

$$
\begin{aligned}
& Q=\int d \sigma\left\{\lambda^{\alpha} p_{\alpha}-\frac{1}{4 \mathcal{T}}\left(\lambda \gamma^{m} \theta\right)\left(P_{m}+\mathcal{T} \partial_{\sigma} X_{m}\right)+\frac{1}{16 \mathcal{T}}\left(\lambda \gamma^{m} \theta\right)\left(\theta \gamma_{m} \partial_{\sigma} \theta\right)\right\}, \\
& \hat{Q}=\int d \sigma\left\{\hat{\lambda}^{\hat{\alpha}} \hat{p}_{\hat{\alpha}}-\frac{1}{4 \mathcal{T}}\left(\hat{\lambda} \gamma^{m} \hat{\theta}\right)\left(P_{m}-\mathcal{T} \partial_{\sigma} X_{m}\right)-\frac{1}{16 \mathcal{T}}\left(\hat{\lambda} \gamma^{m} \hat{\theta}\right)\left(\hat{\theta} \gamma_{m} \partial_{\sigma} \hat{\theta}\right)\right\},
\end{aligned}
$$

Finally, note that the BRST charges and the action (2.72) are spacetime supersymmetric and the supersymmetry generators can be expressed as

$$
\begin{aligned}
& q_{\alpha}=\int d \sigma\left\{p_{\alpha}+\frac{1}{4 \mathcal{T}}\left(P_{m}+\mathcal{T} \partial_{\sigma} X_{m}\right)\left(\gamma^{m} \theta\right)_{\alpha}+\frac{1}{48 \mathcal{T}}\left(\theta \gamma_{m} \partial_{\sigma} \theta\right)\left(\gamma^{m} \theta\right)_{\alpha}\right\} \\
& \hat{q}_{\hat{\alpha}}=\int d \sigma\left\{\hat{p}_{\hat{\alpha}}+\frac{1}{4 \mathcal{T}}\left(P_{m}-\mathcal{T} \partial_{\sigma} X_{m}\right)\left(\gamma^{m} \hat{\theta}\right)_{\hat{\alpha}}-\frac{1}{48 \mathcal{T}}\left(\hat{\theta} \gamma_{m} \partial_{\sigma} \hat{\theta}\right)\left(\gamma^{m} \hat{\theta}\right)_{\hat{\alpha}}\right\}
\end{aligned}
$$

satisfying the algebra

$$
\begin{aligned}
& \left\{q_{\alpha}, q_{\beta}\right\}=\frac{1}{2 \mathcal{T}} \gamma_{\alpha \beta}^{m} \int d \sigma P_{m}, \\
& \left\{\hat{q}_{\hat{\alpha}}, \hat{q}_{\hat{\beta}}\right\}=\frac{1}{2 \mathcal{T}} \gamma_{\hat{\alpha} \hat{\beta}}^{m} \int d \sigma P_{m} .
\end{aligned}
$$

Therefore, spacetime supersymmetry is made manifest with the help of the field redefinitions (2.64) and the pure spinor gauge symmetries (2.71), demonstrating the the action (2.18) can be seen as the underlying gauge theory of the pure spinor superstring. 


\section{Final remarks}

The pure spinor action (2.72) can be rewritten in a more traditional way by solving the equation of motion for $P_{m}$ and Wick-rotating the worldsheet time coordinate $\tau$. The resulting action is

$$
S=\int d^{2} z\left\{\frac{1}{2} \partial X^{m} \bar{\partial} X_{m}+w_{\alpha} \bar{\partial} \lambda^{\alpha}+p_{\alpha} \bar{\partial} \theta^{\alpha}+\hat{w}_{\hat{\alpha}} \partial \hat{\lambda}^{\hat{\alpha}}+\hat{p}_{\hat{\alpha}} \partial \hat{\theta}^{\hat{\alpha}}\right\}
$$

with $z(\bar{z})$ denoting the usual (anti)holomorphic coordinate, $\partial \equiv \frac{\partial}{\partial z}, \bar{\partial} \equiv \frac{\partial}{\partial \bar{z}}$ and $\mathcal{T}=1$ (string tension). The BRST charge (2.74a) takes its standard form in the pure spinor formalism as

$$
Q_{P S}=\oint \lambda^{\alpha} d_{\alpha}
$$

where $d_{\alpha}$ denotes the field realization of the supersymmetric derivative and is expressed as

$$
d_{\alpha} \equiv p_{\alpha}-\frac{1}{2} \partial X^{m}\left(\gamma_{m} \theta\right)_{\alpha}-\frac{1}{8}\left(\theta \gamma^{m} \partial \theta\right)\left(\gamma_{m} \theta\right)_{\alpha}
$$

While the twistor-like symmetry can be understood as a way to rewrite the generators of worldsheet reparametrization with a linear dependence on $P_{m}$, the extra fermionic pair $\left\{\xi^{\alpha}, p_{\alpha}\right\}$ and the fermionic symmetry (2.25) do not have a clear physical interpretation. These ingredients are ultimately responsible for the emergence of spacetime supersymmetry but from the worldsheet point of view their existence lacks a more fundamental understanding. The operator $\lambda^{\alpha} p_{\alpha}$ resembles part of a possible worldsheet supersymmetry generator with "wrong" conformal dimension so it might be possible that the gauge action (2.18) can be embedded in a bigger model with twisted worldsheet supersymmetry. It would be interesting to investigate potential connections with (1) the superembedding origin of the heterotic pure spinor superstring, presented in [20]; and (2) the twisted formulation of the pure spinor superstring introduced in [25] and its relation to the spinning string. Also in this direction, it is worth studying alternative gauge choices for the master action (2.37) and whether they could be related to the Green-Schwarz superstring. This idea was first proposed in [23] for Berkovits' action (1.3) and it would be interesting to develop a similar approach here. In order to do that, it seems that worldsheet reparametrization has to be explicitly included in the construction of the master action.

\section{Acknowledgments}

I would like to thank Thales Azevedo and Nathan Berkovits for comments of the draft. Also, I would like to thank Dmitri Sorokin for reference suggestions and for pointing out a possible connection between the results presented here and the superembedding approach. This research has been supported by the Czech Science Foundation - GAČR, project 1906342Y. 


\section{A Partial gauge fixing of the pure spinor symmetries}

The aim of this appendix is to demonstrate that the action (2.69) can be rewritten in terms of unconstrained spacetime spinors $p_{\alpha}, \theta^{\alpha}, \hat{p}_{\hat{\alpha}}$ and $\hat{\theta}^{\hat{\alpha}}$, provided that the pure spinor gauge symmetries (2.71) are partially fixed in a precise form. In order to do this, the pure spinor constraints (2.70) will be explicitly solved in a Wick-rotated scenario and the $\mathrm{SO}(10)$ spinors will be expressed in terms of $\mathrm{U}(5)$ components. To illustrate the procedure, only the unhatted (left-moving) sector will be analyzed, but it can be easily extended to the right-moving sector.

U(5) decomposition. Given an $S O(10)$ chiral spinor $\xi^{\alpha}$ (antichiral $\chi_{\alpha}$ ), with $\alpha=$ $1, \ldots, 16$, it is possible to determine its $U(5)$ components using the projectors $P_{A}^{\alpha}$ and $\left(P_{A}^{\alpha}\right)^{-1} \equiv P_{\alpha}^{I}$, where $A=\{+, a, a b\}$ denotes the $U(5)$ indices, respectively the $\mathrm{U}(1)$ charged singlet, the vector and the adjoint representations, with $a=1, \ldots, 5$, defined in such a way that

$$
\begin{aligned}
\xi^{\alpha} & =P_{+}^{\alpha} \xi^{+}+\frac{1}{2} P_{a b}^{\alpha} \xi^{a b}+P^{\alpha a} \xi_{a}, & \chi_{\alpha} & =P_{\alpha}^{+} \chi_{+}+\frac{1}{2} P_{\alpha}^{a b} \chi_{a b}+P_{\alpha a} \chi^{a}, \\
\xi^{+} & =P_{\alpha}^{+} \xi^{\alpha}, & \chi_{+} & =P_{+}^{\alpha} \chi_{\alpha}, \\
\xi^{a b} & =P_{\alpha}^{a b} \xi^{\alpha}, & \chi_{a b} & =P_{a b}^{\alpha} \chi_{\alpha}, \\
\xi_{a} & =P_{\alpha a} \xi^{\alpha}, & \chi^{a} & =P^{\alpha a} \chi_{\alpha} .
\end{aligned}
$$

These projectors satisfy the orthogonality equations

$$
\begin{aligned}
P_{+}^{\alpha} P_{\alpha}^{+} & =1, \\
P^{\alpha a} P_{\alpha b} & =\delta_{b}^{a}, \\
P_{a b}^{\alpha} P_{\alpha}^{c d} & =\delta_{a}^{c} \delta_{b}^{d}-\delta_{b}^{c} \delta_{a}^{d} \\
P_{+}^{\alpha} P_{\beta}^{+}+\frac{1}{2} P_{a b}^{\alpha} P_{\beta}^{a b}+P^{\alpha a} P_{\beta a} & =\delta_{\beta}^{\alpha},
\end{aligned}
$$

and more generally $P_{A}^{\alpha} P_{\alpha}^{B}=0$ for $A \neq B$. They can be used as building blocks of the symmetric $g$-matrices, defined as

$$
\begin{aligned}
\left(g^{a}\right)^{\alpha \beta} & \equiv \sqrt{2}\left(P_{+}^{\alpha} P^{\beta a}+P_{+}^{\beta} P^{\alpha a}+\frac{1}{4} \epsilon^{a b c d e} P_{b c}^{\alpha} P_{d e}^{\beta}\right), \\
\left(\bar{g}_{a}\right)^{\alpha \beta} & \equiv \sqrt{2}\left(P^{\alpha b} P_{b a}^{\beta}+P^{\beta b} P_{b a}^{\alpha}\right), \\
\left(g^{a}\right)_{\alpha \beta} & \equiv \sqrt{2}\left(P_{\beta b} P_{\alpha}^{b a}+P_{\alpha b} P_{\beta}^{b a}\right), \\
\left(\bar{g}_{a}\right)_{\alpha \beta} & \equiv \sqrt{2}\left(P_{\alpha}^{+} P_{\beta a}+P_{\beta}^{+} P_{\alpha a}+\frac{1}{4} \epsilon_{a b c d e} P_{\alpha}^{b c} P_{\beta}^{d e}\right),
\end{aligned}
$$

where $\epsilon^{a b c d e}$ and $\epsilon_{a b c d e}$ are the totally antisymmetric $U(5)$ tensors, with $\epsilon^{12345}=\epsilon_{54321}=1$, satisfying the algebra

$$
\begin{aligned}
\left\{g^{a}, g^{b}\right\}_{\alpha}{ }^{\beta} & \equiv g_{\alpha \gamma}^{a}\left(g^{b}\right)^{\gamma \beta}+g_{\alpha \gamma}^{b}\left(g^{a}\right)^{\gamma \beta} \\
& =0, \\
\left\{\bar{g}_{a}, \bar{g}_{b}\right\}_{\alpha}{ }^{\beta} & \equiv\left(\bar{g}_{a}\right)_{\alpha \gamma} \bar{g}_{b}^{\gamma \beta}+\left(\bar{g}_{b}\right)_{\alpha \gamma} \bar{g}_{a}^{\gamma \beta} \\
& =0, \\
\left\{g^{a}, \bar{g}_{b}\right\}_{\alpha}{ }^{\beta} & \equiv g_{\alpha \gamma}^{a} \bar{g}_{b}^{\gamma \beta}+\left(\bar{g}_{b}\right)_{\alpha \gamma}\left(g^{a}\right)^{\gamma \beta} \\
& =2 \delta^{\beta} \delta_{b}^{a} .
\end{aligned}
$$

Therefore, the $g$-matrices (A.3) represent the $\mathrm{U}(5)$ components of the gamma matrices $\gamma^{m}$. 
Solving the pure spinor constraints. The action (2.69) is invariant under the pure spinor gauge transformations

$$
\begin{aligned}
\delta w_{\alpha} & =d_{m}\left(\gamma^{m} \lambda\right)_{\alpha}+e_{m}\left(\gamma^{m} \xi\right)_{\alpha}-\bar{f}_{m n}\left(\gamma^{m n} \pi\right)_{\alpha} \\
\delta p_{\alpha} & =e_{m}\left(\gamma^{m} \lambda\right)_{\alpha} \\
\delta \theta^{\alpha} & =\bar{f}_{m n}\left(\gamma^{m n} \lambda\right)^{\alpha}
\end{aligned}
$$

where $d_{m}, e_{m}$ and $\bar{f}_{m n}$ are the parameters. Note that the parameters $\bar{f}$ and $\bar{g}$ in $(2.71)$ are redundant, for they can be absorbed by a shift of the other parameters:

$$
\begin{aligned}
d_{m} & \rightarrow d_{m}-\frac{4 \bar{f}}{5} \frac{\left(\Lambda \gamma_{m} \pi\right)}{(\Lambda \lambda)}+\frac{\bar{g}}{5} \frac{(\Lambda \xi)}{(\Lambda \lambda)^{2}}\left(\Lambda \gamma_{m} \pi\right) \\
e_{m} & \rightarrow e_{m}+\frac{\bar{g}}{2} \frac{\left(\Lambda \gamma_{m} \pi\right)}{(\Lambda \lambda)} \\
\bar{f}_{m n} & \rightarrow \bar{f}_{m n}+\frac{\bar{f}}{10} \frac{\left(\Lambda \gamma_{m n} \lambda\right)}{(\Lambda \lambda)}+\frac{\bar{g}}{8} \frac{\left(\Lambda \gamma_{m n} \xi\right)}{(\Lambda \lambda)}-\frac{\bar{g}}{40} \frac{(\Lambda \xi)\left(\Lambda \gamma_{m n} \lambda\right)}{(\Lambda \lambda)^{2}}
\end{aligned}
$$

This can be easily demonstrated with the help of the gamma matrix property (2.58).

In terms of $\mathrm{U}(5)$ components, $\lambda^{\alpha}, \theta^{\alpha}$ and $\pi_{\alpha}$ can be parametrized as $\lambda^{\alpha}=\left(\lambda^{+}, \lambda^{a b}, \lambda_{a}\right)$, $\xi^{\alpha}=\left(\xi^{+}, \xi^{a b}, \xi_{a}\right)$, and $\pi_{\alpha}=\left(\pi_{+}, \pi_{a b}, \pi^{a}\right)$. Therefore, using the $g$-matrices (A.3), the constraints (2.70) can be rewritten as

$$
\begin{aligned}
\lambda^{a b} \lambda_{b} & =0, & \lambda^{+} \pi_{a b} & =\frac{1}{2} \epsilon_{a b c d e} \pi^{c} \lambda^{d e}, \\
\lambda^{+} \lambda_{a} & =-\frac{1}{8} \epsilon_{a b c d e} \lambda^{b c} \lambda^{d e}, & \lambda^{+} \pi_{+}- & =\frac{3}{5} \lambda_{a} \pi^{a}-\frac{1}{10} \lambda^{a b} \pi_{a b}, \\
\lambda^{a b} \xi_{b} & =-\xi^{a b} \lambda_{b}, & \lambda_{a} \pi^{b}-\lambda^{b c} \pi_{a c} & =\frac{1}{5} \delta_{a}^{b}\left(\lambda_{c} \pi^{c}-\lambda^{c d} \pi_{c d}\right), \\
\lambda^{+} \xi_{a} & =-\xi^{+} \lambda_{a}-\frac{1}{4} \epsilon_{a b c d e} \lambda^{b c} \xi^{d e}, & \lambda^{+} \pi_{+}- & =\lambda_{a} \pi^{a}+\frac{1}{2} \lambda^{a b} \pi_{a b}, \\
\lambda^{a b} \pi_{+} & =\frac{1}{2} \epsilon^{a b c d e} \lambda_{c} \pi_{d e}, & \xi^{+} \pi_{+}- & =\xi_{a} \pi^{a}+\frac{1}{2} \xi^{a b} \pi_{a b} .
\end{aligned}
$$

Assuming $\lambda^{+} \neq 0$, these constraints can be explicitly solved by

$$
\begin{aligned}
\lambda_{a} & =-\frac{1}{8 \lambda^{+}} \epsilon_{a b c d e} \lambda^{b c} \lambda^{d e}, \\
\xi_{a} & =-\frac{\xi^{+}}{\lambda^{+}} \lambda_{a}-\frac{1}{4 \lambda^{+}} \epsilon_{a b c d e} \lambda^{b c} \xi^{d e}, \\
\pi_{+} & =\frac{1}{\lambda^{+}} \lambda_{a} \pi^{a} \\
\pi_{a b} & =\frac{1}{2 \lambda^{+}} \epsilon_{a b c d e} \pi^{c} \lambda^{d e}
\end{aligned}
$$

Next, the transformations (A.5) can be used to conveniently choose the gauge $p_{\alpha}=$ $\left\{p_{+}, p_{a b}, \pi^{a}\right\}$ and $\theta^{\alpha}=\left\{\xi^{+}, \xi^{a b}, \theta_{a}\right\}$, where $p_{+}, p_{a b}$ and $\theta_{a}$ denote the independent components of $p_{\alpha}$ and $\theta^{\alpha}$. Using this gauge and the solutions (A.8), their contribution to the action (2.69) can be cast as

$$
\begin{aligned}
S_{s p}= & \int d \tau d \sigma\left\{p_{\alpha} \partial_{-} \xi^{\alpha}+\pi_{\alpha} \partial_{-} \theta^{\alpha}\right\} \\
= & \int d \tau d \sigma\left\{p_{+} \partial_{-} \xi^{+}+\frac{1}{2} p_{a b} \partial_{-} \xi^{a b}+p^{a} \partial_{-} \xi_{a}\right\} \\
& +\int d \tau d \sigma\left\{\pi_{+} \partial_{-} \theta^{+}+\frac{1}{2} \pi_{a b} \partial_{-} \theta^{a b}+\pi^{a} \partial_{-} \theta_{a}\right\}, \\
= & \int d \tau d \sigma\left\{p_{\alpha} \partial_{-} \theta^{\alpha}-\pi^{a} \xi^{+} \partial_{-}\left(\frac{\lambda_{a}}{\lambda^{+}}\right)-\frac{1}{4} \epsilon_{a b c d e} \pi^{a} \xi^{d e} \partial_{-}\left(\frac{\lambda^{b c}}{\lambda^{+}}\right)\right\} .
\end{aligned}
$$


Note that the last two terms can be absorbed by a redefinition of $w_{\alpha}$ and the resulting action (2.72) depends only on the unconstrained pair $\left\{p_{\alpha}, \theta^{\alpha}\right\}$.

Furthermore, the BRST current derived from the action (2.74a) is rewritten as well in terms of the pair $\left\{p_{\alpha}, \theta^{\alpha}\right\}$. The terms $\lambda^{\alpha} p_{\alpha}$ and $\left(\lambda \gamma^{m} \theta\right)$ preserve their shape with the gauge choice above and after a simple similarity transformation, the BRST current is given by

$$
J=\lambda^{\alpha} p_{\alpha}-\frac{1}{4 \mathcal{T}}\left(\lambda \gamma_{m} \theta\right)\left[P^{m}+\mathcal{T} \partial_{\sigma} X^{m}+\frac{1}{4}\left(\theta \gamma^{m} \partial_{\sigma} \theta\right)\right]
$$

corresponding to the usual pure spinor BRST current plus a U(1) decoupled sector.

\section{B The $\mathrm{U}(1)_{R} \times \mathrm{U}(1)_{L}$ sector}

This section presents some properties of the $\mathrm{U}(1)_{R} \times \mathrm{U}(1)_{L}$ sector, which is constituted by the ghosts coming from the scaling symmetry (2.24) and the fermionic symmetry (2.25), but after the field redefinition (2.64).

After a Wick-rotation of the worldsheet time $\tau$, their contribution to the action (2.60) can be written as

$$
S_{*}=\int d^{2} z\{\bar{\Omega} \bar{\partial} \Omega+\beta \bar{\partial} \gamma+\hat{\bar{\Omega}} \partial \hat{\Omega}+\hat{\beta} \partial \hat{\gamma}\}
$$

with holomorphic and anti-holomorphic BRST currents given by

$$
\begin{aligned}
& J_{*}=\Omega\left(\frac{1}{2} \partial \ln \gamma-\beta \gamma\right), \\
& \hat{J}_{*}=\hat{\Omega}\left(\frac{1}{2} \bar{\partial} \ln \hat{\gamma}-\hat{\beta} \hat{\gamma}\right) .
\end{aligned}
$$

Note that the constants $c_{\#}$ and $\hat{c}_{\#}$ in (2.65) were fixed by requiring nilpotency of the BRST charges $Q_{*} \equiv \oint J_{*}$ and $\hat{Q}_{*} \equiv \oint \hat{J}_{*}$.

The cohomology of $Q_{*}$ has only two elements, the identity operator $\mathbb{1}$ and $\Omega$, which is BRST singular,

$$
\Omega=\lim _{\epsilon \rightarrow 0} \frac{1}{\epsilon}\left[Q_{*}, \gamma^{\epsilon}\right]
$$

Any other BRST-closed operator can be shown to be $Q_{*}$-exact and there is no operator trivializing the cohomology.

Because of the field redefinition (2.64), $\beta$ and $\hat{\beta}$ are not conformal primary operators. In fact, the components of the energy-momentum tensor have a non-standard form given by

$$
\begin{aligned}
& T_{*}=-\bar{\Omega} \partial \Omega-\beta \partial \gamma+\frac{1}{2 \gamma^{2}}\left[\gamma \partial^{2} \gamma-(\partial \gamma)^{2}\right], \\
& \hat{T}_{*}=-\hat{\bar{\Omega}} \bar{\partial} \hat{\Omega}-\hat{\beta} \bar{\partial} \hat{\gamma}+\frac{1}{2 \hat{\gamma}^{2}}\left[\hat{\gamma} \bar{\partial}^{2} \hat{\gamma}-(\bar{\partial} \hat{\gamma})^{2}\right] .
\end{aligned}
$$

Using the fundamental OPE

$$
\gamma(z) \beta(y) \sim \frac{1}{(z-y)}
$$


the following results are obtained

$$
\begin{aligned}
T_{*}(z) \gamma(y) & \sim \frac{\partial \gamma}{(z-y)}, \\
T_{*}(z) \beta(y) & \sim \frac{\gamma^{-1}}{(z-y)^{3}}+\frac{\beta}{(z-y)^{2}}+\frac{\partial \beta}{(z-y)}, \\
T_{*}(z) \Omega(y) & \sim \frac{\partial \Omega}{(z-y)}, \\
T_{*}(z) \bar{\Omega}(y) & \sim \frac{\bar{\Omega}}{(z-y)^{2}}+\frac{\partial \bar{\Omega}}{(z-y)}, \\
T_{*}(z) J_{*}(y) & \sim \frac{J_{*}}{(z-y)^{2}}+\frac{\partial J_{*}}{(z-y)}, \\
T_{*}(z) T_{*}(y) & \sim \frac{2 T_{*}}{(z-y)^{2}}+\frac{\partial T_{*}}{(z-y)} .
\end{aligned}
$$

Apart from $\beta$, all the other operators above are primary fields and the central charge of the model vanishes.

As discussed in the main text, the ghost $\gamma$ should not have any zeros on the worldsheet, otherwise the field redefinitions (2.64) are ill defined. This condition can be enforced by the parametrization

$$
\begin{aligned}
& \gamma=e^{\sigma}, \\
& \beta=\rho e^{-\sigma},
\end{aligned}
$$

and similarly for the hatted sector, such that the holomorphic part of the action (B.1) and its associated BRST charge are given by

$$
\begin{aligned}
S_{*} & =\int d^{2} z\{\bar{\Omega} \bar{\partial} \Omega+\rho \bar{\partial} \sigma\}, \\
Q_{*} & =\oint \Omega \rho .
\end{aligned}
$$

Using the quartet argument, it is easy to show that the cohomology of $Q_{*}$ contains only one element, the identity operator.

\section{A non-minimal formalism with fundamental $(b, c)$ ghosts}

Because worldsheet reparametrization is a redundant symmetry of the action (2.18), the gauge fixed model does not contain the fundamental $(b, c)$ ghosts which are directly connected to the definition of perturbative string theory as a sum over different worldsheet topologies. In order to implement such structure in the pure spinor formalism, Berkovits proposed the composite $b$ ghost in [26], which was later made super Poincarè covariant with the introduction of the non-minimal formalism in [27]. In its simplest form [28], the pure spinor composite $b$ ghost can be defined classically as

$$
B \equiv \frac{\Lambda_{\alpha}}{4(\Lambda \lambda)}\left(2 \partial X^{m}\left(\gamma_{m} d\right)^{\alpha}+\left(\theta \gamma^{m} \partial \theta\right)\left(\gamma_{m} d\right)^{\alpha}+\frac{1}{2}\left(w \gamma_{m n} \lambda\right)\left(\gamma^{m n} \partial \theta\right)^{\alpha}+(w \lambda) \partial \theta^{\alpha}\right),
$$


and satisfies $\{Q, B\}=T_{P S}$, where $Q$ is the BRST charge (3.2) and $T_{P S}$ denotes the holomorphic component of the energy-momentum tensor associated to (3.1) and can be expressed as

$$
T_{P S}=-\frac{1}{2} \partial X^{m} \partial X_{m}-p_{\alpha} \partial \theta^{\alpha}-w_{\alpha} \partial \lambda^{\alpha} .
$$

When the master action (2.37) is extended to include all the gauge and gauge-forgauge symmetries described in subsection (2.3), it is possible to choose a gauge in which the reparametrization ghosts survive in the form of non-minimal variables such that the resulting BRST charge can be expressed as

$$
Q=e^{-U}\left(\oint\left\{\lambda^{\alpha} d_{\alpha}+b \phi\right\}\right) e^{U}
$$

where $U$ is the generator of a similarity transformation given by

$$
U=\oint\{-c B+\bar{\phi} c \partial c\} .
$$

Here, $b, c, \bar{\phi}$ and $\phi$ are the (fundamental) Virasoro ghosts and ghost-for-ghosts with vanishing contribution to the central charge of the action $(-26+26=0)$.

The BRST charge (C.3) has the structure of a generic coupling of the pure spinor superstring to topological two-dimensional gravity, as analyzed in [29], and was already suggested in [22], but it will be further explored here. First note that it can be rewritten as

$$
Q=\oint\left\{\lambda^{\alpha} d_{\alpha}+c\left(T_{P S}-2 \bar{\phi} \partial \phi-\phi \partial \bar{\phi}-b \partial c\right)+b \phi\right\}
$$

such that the fundamental $b$ ghost satisfies

$$
\{Q, b\}=T
$$

where $T$ is the energy-momentum tensor of the non-minimal action,

$$
T=T_{P S}-\bar{\phi} \partial \phi-\partial(\bar{\phi} \phi)-b \partial c-\partial(b c) .
$$

Therefore, the similarity transformation in (C.3) helps to expose the familiar construction from gauge fixing worldsheet diffeomorphisms. On the other hand, there does not seem to be any relevant advantage in making such structure manifest.

The whole machinery related to picture changing operators can be immediately built. The bosonic ghosts $\phi$ and $\bar{\phi}$ can be fermionized as follows,

$$
\begin{aligned}
& \phi \cong e^{\sigma} \eta, \\
& \bar{\phi} \cong e^{-\sigma} \partial \xi
\end{aligned}
$$

such that the BRST cohomology of (C.3) can be written at picture -1 as

$$
V^{(-1)}=e^{-U}\left(c e^{-\sigma} V\right) e^{U},
$$


where $V$ is an element of the pure spinor cohomology, with BRST charge (3.2). Integrated vertex operators, $I$, can be built with the action of the fundamental $b$ ghost on the unintegrated vertex operators. In the picture -1 , they can be built as

$$
\begin{aligned}
I^{(-1)} & \equiv \oint b \cdot V^{(-1)}, \\
& =e^{-U}\left(\oint\left\{e^{-\sigma} V+\ldots\right\}\right) e^{U} .
\end{aligned}
$$

Picture changing operators are also defined in a simple way. The picture raising operator is given by

$$
\begin{aligned}
Y & \equiv\{Q, \xi\} \\
& =c \partial \xi+(b-B) e^{\sigma},
\end{aligned}
$$

while the picture lowering is given by

$$
Z \equiv c e^{-\sigma}
$$

such that

$$
\lim _{z \rightarrow y} Z(z) Y(y)=1 .
$$

Note that because of the manifest spacetime supersymmetry, Neveu-Schwarz and Ramond states are treated on equal footing and there are no half-integer pictures.

At tree level, the integration measure for the reparametrization ghosts and ghost-forghosts is simply

$$
\left\langle c \partial c \partial^{2} c e^{-3 \sigma}\right\rangle=1 .
$$

$N$-point amplitudes can then be computed with 3 vertices with picture -1 to saturate the background charge $(\sigma)$ and $(N-3)$ vertices with picture 0 .

In order to have a fully covariant formulation of this model, the composite $B$ ghost introduced by Berkovits in [27] can be used. Observe that for higher genus, $B$ will enter through the picture changing operator, so it agrees with the usual pure spinor prescription in which the $B$ ghost helps to saturate the number of fermionic fields $\left(d_{\alpha}\right)$.

\section{The sectorized and ambitwistor pure spinor superstrings}

The gauge (2.46) relies on the connection between the twistor-like constraints and the generators of worldsheet reparametrization in the first order formalism, represented through the identifications displayed in (2.15). As mentioned earlier, the gauge $L^{\alpha}=\hat{L}^{\hat{\alpha}}=0$ would imply a degenerate worldsheet metric. To see this, consider a different gauge fixing fermion of the form

$$
\Xi=\int d \tau d \sigma\left\{\bar{\Omega} A_{\tau}+\beta \chi_{\tau}-r^{\alpha} w_{\alpha}^{\sigma}+\eta^{\alpha} p_{\alpha}^{\sigma}-\pi_{\alpha} L^{\alpha}+\hat{\bar{\Omega}} \hat{A}_{\tau}-\hat{r}^{\hat{\alpha}} \hat{w}_{\hat{\alpha}}^{\sigma}+\hat{\beta} \hat{\chi}_{\tau}+\hat{\eta}^{\hat{\alpha}} \hat{p}_{\hat{\alpha}}^{\sigma}-\hat{\pi}_{\hat{\alpha}} \hat{L}^{\hat{\alpha}}\right\},
$$


thus implementing the singular gauge. The gauge fixed action can then be shown to be

$$
\begin{aligned}
\tilde{S}= & \int d \tau d \sigma\left\{P_{m} \partial_{\tau} X^{m}+w_{\alpha} \partial_{\tau} \lambda^{\alpha}+p_{\alpha} \partial_{\tau} \xi^{\alpha}+\pi_{\alpha} \partial_{\tau} \theta^{\alpha}+r^{\alpha} \partial_{\tau} s_{\alpha}+\eta^{\alpha} \partial_{\tau} \epsilon_{\alpha}+A_{\sigma} \partial_{\tau} B\right\} \\
& +\int d \tau d \sigma\left\{\hat{w}_{\hat{\alpha}} \partial_{\tau} \hat{\lambda}^{\hat{\alpha}}+\hat{p}_{\hat{\alpha}} \partial_{\tau} \hat{\xi}^{\hat{\alpha}}+\hat{\pi}_{\hat{\alpha}} \partial_{\tau} \hat{\theta}^{\hat{\alpha}}+\hat{r}^{\hat{\alpha}} \partial_{\tau} \hat{s}_{\hat{\alpha}}+\hat{\eta}^{\hat{\alpha}} \partial_{\tau} \hat{\epsilon}_{\hat{\alpha}}+\hat{A}_{\sigma} \partial_{\tau} \hat{B}\right\} \\
& +\int d \tau d \sigma\left\{\Sigma \partial_{\tau} \chi_{\sigma}+\bar{\Omega} \partial_{\tau} \Omega+\beta \partial_{\tau} \gamma+\hat{\Sigma} \partial_{\tau} \hat{\chi}_{\sigma}+\hat{\bar{\Omega}}_{\tau} \hat{\Omega}+\hat{\beta} \partial_{\tau} \hat{\gamma}\right\}
\end{aligned}
$$

The problem with this action is a residual gauge symmetry (aside from the pure spinor symmetries) of the form $\delta \tau \rightarrow f(\tau)$, a reparametrization of the worldsheet time coordinate. Therefore, the singular gauge proposed in [23] does not completely fix the gauge symmetries of the action (2.18).

There is, however, another interesting singular gauge. Consider now the gauge fixing fermion

$$
\begin{aligned}
\Xi= & \int d \tau d \sigma\left\{\bar{\Omega} A_{\tau}+\beta \chi_{\tau}-r^{\alpha} w_{\alpha}^{\sigma}+\eta^{\alpha} p_{\alpha}^{\sigma}+\hat{\bar{\Omega}} \hat{A}_{\tau}-\hat{r}^{\hat{\alpha}} \hat{w}_{\hat{\alpha}}^{\sigma}+\hat{\beta} \hat{\chi}_{\tau}+\hat{\eta}^{\hat{\alpha}} \hat{p}_{\hat{\alpha}}^{\sigma}\right\} \\
& -\int d \tau d \sigma\left\{\pi_{\alpha}\left[L^{\alpha}-\frac{\left(\gamma^{m} \Lambda\right)^{\alpha}}{(\Lambda \lambda)}\left(P_{m}+\mathcal{T} \partial_{\sigma} X_{m}\right)\right]+\hat{\pi}_{\hat{\alpha}}\left[\hat{L}^{\hat{\alpha}}+\frac{\left(\gamma^{m} \hat{\Lambda}\right)^{\hat{\alpha}}}{(\hat{\Lambda} \hat{\lambda})}\left(P_{m}-\mathcal{T} \partial_{\sigma} X_{m}\right)\right]\right\},
\end{aligned}
$$

which differs from (2.47) by a sign in the last term. In an analogy with the first order Polyakov action (2.7), this gauge can be interpreted as $e_{+}=-e_{-}=1$, cf. (2.15). ${ }^{2}$ In terms of the worldsheet metric, (2.6), this gauge is equivalent to $g_{\tau \tau}=0$. The gauge fixed action, after the addition of a BRST expression, similarly to what is described in subsection (2.5), can be written as

$$
\begin{aligned}
\tilde{S}= & \int d \tau d \sigma\left\{P_{m} \partial_{-} X^{m}+w_{\alpha} \partial_{-} \lambda^{\alpha}+p_{\alpha} \partial_{-} \xi^{\alpha}+A \partial_{-} B+\hat{w}_{\hat{\alpha}} \partial_{-} \hat{\lambda}^{\hat{\alpha}}+\hat{p}_{\hat{\alpha}} \partial_{-} \hat{\xi}^{\hat{\alpha}}+\hat{A} \partial_{-} \hat{B}\right\} \\
& +\int d \tau d \sigma\left\{\pi_{\alpha} \partial_{-} \theta^{\alpha}+r^{\alpha} \partial_{-} s_{\alpha}+\eta^{\alpha} \partial_{-} \epsilon_{\alpha}+\hat{\pi}_{\hat{\alpha}} \partial_{-} \hat{\theta}^{\hat{\alpha}}+\hat{r}^{\hat{\alpha}} \partial_{-} \hat{s}_{\hat{\alpha}}+\hat{\eta}^{\hat{\alpha}} \partial_{-} \hat{\epsilon}_{\hat{\alpha}}\right\} \\
& +\int d \tau d \sigma\left\{\Sigma \partial_{-} \chi+\bar{\Omega} \partial_{-} \Omega+\beta \partial_{-} \gamma+\hat{\Sigma} \partial_{-} \hat{\chi}+\hat{\bar{\Omega}} \partial_{-} \hat{\Omega}+\hat{\beta} \partial_{-} \hat{\gamma}\right\}
\end{aligned}
$$

and all the worldsheet fields satisfy the equation of motion $\partial_{-}=0$, constituting a chiral model. Furthermore, following the procedure described in subsection (2.6), the resulting action is given by

$$
S=\int d \tau d \sigma\left\{P_{m} \partial_{-} X^{m}+w_{\alpha} \partial_{-} \lambda^{\alpha}+p_{\alpha} \partial_{-} \theta^{\alpha}+\hat{w}_{\hat{\alpha}} \partial_{-} \hat{\lambda}^{\hat{\alpha}}+\hat{p}_{\hat{\alpha}} \partial_{-} \hat{\theta}^{\hat{\alpha}}\right\}
$$

which corresponds to the sectorized string introduced in [30]. The two chiral components of the BRST current can be cast as

$$
\begin{aligned}
& J=\lambda^{\alpha} p_{\alpha}-\frac{1}{4 \mathcal{T}}\left(\lambda \gamma_{m} \theta\right)\left(P^{m}+\frac{\mathcal{T}}{2} \partial_{+} X^{m}\right)+\frac{1}{32 \mathcal{T}}\left(\lambda \gamma_{m} \theta\right)\left(\theta \gamma^{m} \partial_{+} \theta\right), \\
& \hat{J}=\hat{\lambda}^{\hat{\alpha}} \hat{p}_{\hat{\alpha}}-\frac{1}{4 \mathcal{T}}\left(\hat{\lambda} \gamma_{m} \hat{\theta}\right)\left(P^{m}-\frac{\mathcal{T}}{2} \partial_{+} X^{m}\right)-\frac{1}{32 \mathcal{T}}\left(\hat{\lambda} \gamma_{m} \hat{\theta}\right)\left(\hat{\theta} \gamma^{m} \partial_{+} \hat{\theta}\right) .
\end{aligned}
$$

\footnotetext{
${ }^{2}$ I would like to thank Thales Azevedo for observing this is the gauge choice leading to the bosonic sectorized model.
} 
As a consequence of the singular gauge choice, the string tension disappears from the action (D.5), although it is still present in the BRST current. Note also that the spacetime spinors can be made dimensionless via a scale transformation of the form

$$
\begin{aligned}
\lambda^{\alpha} \rightarrow \mathcal{T}^{1 / 2} \lambda^{\alpha}, & \theta^{\alpha} \rightarrow \mathcal{T}^{1 / 2} \theta^{\alpha}, \\
w_{\alpha} \rightarrow \mathcal{T}^{-1 / 2} w_{\alpha}, & p_{\alpha} \rightarrow \mathcal{T}^{-1 / 2} p_{\alpha},
\end{aligned}
$$

and similarly for the hatted fields.

Now, the model has a well defined tensionless limit and the BRST currents (D.6) are given by

$$
\begin{aligned}
& J=\lambda^{\alpha} p_{\alpha}-\frac{1}{4}\left(\lambda \gamma^{m} \theta\right) P_{m}, \\
& \hat{J}=\hat{\lambda}^{\hat{\alpha}} \hat{p}_{\hat{\alpha}}-\frac{1}{4}\left(\hat{\lambda} \gamma^{m} \hat{\theta}\right) P_{m}
\end{aligned}
$$

which correspond to the ambitwistor pure spinor superstring [31], in agreement with the results of [32].

The heterotic sectorized or ambitwistor strings in the pure spinor formalism are obtained in a similar way. Worldsheet reparametrization and only one of the twistor-like constraints has to be taken into account in building the master action (2.37). Apart from that, the gauge fixing procedure should be very similar.

Open Access. This article is distributed under the terms of the Creative Commons Attribution License (CC-BY 4.0), which permits any use, distribution and reproduction in any medium, provided the original author(s) and source are credited.

\section{References}

[1] N. Berkovits, Super Poincaré covariant quantization of the superstring, JHEP 04 (2000) 018 [hep-th/0001035] [INSPIRE].

[2] H. Gomez and C.R. Mafra, The closed-string 3-loop amplitude and S-duality, JHEP 10 (2013) 217 [arXiv:1308.6567] [INSPIRE].

[3] C.R. Mafra and O. Schlotterer, Towards the n-point one-loop superstring amplitude. Part I. Pure spinors and superfield kinematics, JHEP 08 (2019) 090 [arXiv:1812.10969] [INSPIRE].

[4] C.R. Mafra and O. Schlotterer, Towards the n-point one-loop superstring amplitude. Part II. Worldsheet functions and their duality to kinematics, JHEP 08 (2019) 091 [arXiv: 1812.10970] [INSPIRE].

[5] C.R. Mafra and O. Schlotterer, Towards the n-point one-loop superstring amplitude. Part III. One-loop correlators and their double-copy structure, JHEP 08 (2019) 092 [arXiv: 1812.10971] [INSPIRE].

[6] L. Mazzucato, Superstrings in AdS, Phys. Rept. 521 (2012) 1 [arXiv:1104.2604] [INSPIRE].

[7] N. Berkovits and E. Witten, Supersymmetry Breaking Effects using the Pure Spinor Formalism of the Superstring, JHEP 06 (2014) 127 [arXiv:1404.5346] [INSPIRE].

[8] P. Ramond, Dual Theory for Free Fermions, Phys. Rev. D 3 (1971) 2415 [InSPIRE]. 
[9] A. Neveu and J.H. Schwarz, Factorizable dual model of pions, Nucl. Phys. B 31 (1971) 86 [INSPIRE].

[10] M.B. Green and J.H. Schwarz, Supersymmetrical Dual String Theory, Nucl. Phys. B 181 (1981) 502 [inSPIRE].

[11] M.B. Green and J.H. Schwarz, Supersymmetrical String Theories, Phys. Lett. 109B (1982) 444 [INSPIRE].

[12] N. Berkovits and C.R. Mafra, Equivalence of two-loop superstring amplitudes in the pure spinor and RNS formalisms, Phys. Rev. Lett. 96 (2006) 011602 [hep-th/0509234] [INSPIRE].

[13] N. Berkovits, Cohomology in the pure spinor formalism for the superstring, JHEP 09 (2000) 046 [hep-th/0006003] [INSPIRE].

[14] N. Berkovits and D.Z. Marchioro, Relating the Green-Schwarz and pure spinor formalisms for the superstring, JHEP 01 (2005) 018 [hep-th/0412198] [INSPIRE].

[15] N. Berkovits and R. Lipinski Jusinskas, Light-Cone Analysis of the Pure Spinor Formalism for the Superstring, JHEP 08 (2014) 102 [arXiv:1406.2290] [INSPIRE].

[16] R.L. Jusinskas, Spectrum generating algebra for the pure spinor superstring, JHEP 10 (2014) 022 [arXiv: 1406.1902] [INSPIRE].

[17] D.P. Sorokin, Superbranes and superembeddings, Phys. Rept. 329 (2000) 1 [hep-th/9906142] [INSPIRE].

[18] N. Berkovits, A Covariant Action for the Heterotic Superstring With Manifest Space-time Supersymmetry and World Sheet Superconformal Invariance, Phys. Lett. B 232 (1989) 184 [INSPIRE].

[19] M. Tonin, World sheet supersymmetric formulations of Green-Schwarz superstrings, Phys. Lett. B 266 (1991) 312 [INSPIRE].

[20] M. Matone, L. Mazzucato, I. Oda, D. Sorokin and M. Tonin, The Superembedding origin of the Berkovits pure spinor covariant quantization of superstrings,

Nucl. Phys. B 639 (2002) 182 [hep-th/0206104] [INSPIRE].

[21] N. Berkovits, Pure spinors, twistors and emergent supersymmetry, JHEP 12 (2012) 006 [arXiv: 1105.1147] [INSPIRE].

[22] N. Berkovits, Twistor Origin of the Superstring, JHEP 03 (2015) 122 [arXiv:1409.2510] [INSPIRE].

[23] N. Berkovits, Origin of the Pure Spinor and Green-Schwarz Formalisms, JHEP 07 (2015) 091 [arXiv: 1503.03080] [INSPIRE].

[24] R.L. Jusinskas, Quantization of the particle with a linear massless solution, arXiv: 1808.07463 [INSPIRE].

[25] N. Berkovits, Untwisting the pure spinor formalism to the RNS and twistor string in a flat and $A d S_{5} \times S^{5}$ background, JHEP 06 (2016) 127 [arXiv:1604.04617] [INSPIRE].

[26] N. Berkovits, Relating the RNS and pure spinor formalisms for the superstring, JHEP 08 (2001) 026 [hep-th/0104247] [INSPIRE].

[27] N. Berkovits, Pure spinor formalism as an $N=2$ topological string, JHEP 10 (2005) 089 [hep-th/0509120] [INSPIRE]. 
[28] I. Oda and M. Tonin, Y-formalism in pure spinor quantization of superstrings, Nucl. Phys. B 727 (2005) 176 [hep-th/0505277] [INSPIRE].

[29] J. Hoogeveen and K. Skenderis, BRST quantization of the pure spinor superstring, JHEP 11 (2007) 081 [arXiv:0710.2598] [INSPIRE].

[30] R.L. Jusinskas, Notes on the ambitwistor pure spinor string, JHEP 05 (2016) 116 [arXiv: 1604.02915] [INSPIRE].

[31] N. Berkovits, Infinite Tension Limit of the Pure Spinor Superstring, JHEP 03 (2014) 017 [arXiv: 1311.4156] [INSPIRE].

[32] T. Azevedo and R.L. Jusinskas, Connecting the ambitwistor and the sectorized heterotic strings, JHEP 10 (2017) 216 [arXiv:1707.08840] [INSPIRE]. 